\title{
Infinitely many sign-changing and semi-nodal solutions for a nonlinear Schrödinger system
}

\author{
ZhiJie Chen, Chang-Shou Lin And Wenming Zou
}

\begin{abstract}
We study the following coupled Schrödinger equations which have appeared as several models from mathematical physics:

$$
\begin{cases}-\Delta u_{1}+\lambda_{1} u_{1}=\mu_{1} u_{1}^{3}+\beta u_{1} u_{2}^{2} & x \in \Omega \\ -\Delta u_{2}+\lambda_{2} u_{2}=\mu_{2} u_{2}^{3}+\beta u_{1}^{2} u_{2} & x \in \Omega \\ u_{1}=u_{2}=0 & \text { on } \partial \Omega .\end{cases}
$$

Here $\Omega$ is a smooth bounded domain in $\mathbb{R}^{N}(N=2,3)$ or $\Omega=\mathbb{R}^{N}, \lambda_{1}, \lambda_{2}$, $\mu_{1}, \mu_{2}$ are all positive constants and the coupling constant $\beta<0$. We show that this system has infinitely many sign-changing solutions. We also obtain infinitely many semi-nodal solutions in the following sense: one component changes sign and the other one is positive. The crucial idea of our proof, which has never been used for this system before, is to study a new problem with two constraints. Finally, when $\Omega$ is a bounded domain, we show that this system has a least energy sign-changing solution, both two components of which have exactly two nodal domains, and we also study the asymptotic behavior of solutions as $\beta \rightarrow-\infty$ and phase separation is expected.
\end{abstract}

Mathematics Subject Classification (2010): 35J20 (primary); 35J50, 35J60 (secondary).

\section{Introduction}

In this paper we study solitary wave solutions of the coupled Gross-Pitaevskii equations $(c f .[8])$ :

$$
\begin{cases}-i \frac{\partial}{\partial t} \Phi_{1}=\Delta \Phi_{1}+\mu_{1}\left|\Phi_{1}\right|^{2} \Phi_{1}+\beta\left|\Phi_{2}\right|^{2} \Phi_{1} & x \in \Omega \quad t>0 \\ -i \frac{\partial}{\partial t} \Phi_{2}=\Delta \Phi_{2}+\mu_{2}\left|\Phi_{2}\right|^{2} \Phi_{2}+\beta\left|\Phi_{1}\right|^{2} \Phi_{2} & x \in \Omega \quad t>0 \\ \Phi_{j}=\Phi_{j}(x, t) \in \mathbb{C} & j=1,2 \\ \Phi_{j}(x, t)=0 & x \in \partial \Omega \quad t>0 \quad j=1,2,\end{cases}
$$

Zou is supported by NSFC $(11025106,11371212,11271386)$ and the Both-Side Tsinghua Fund. Received January 7, 2014; accepted in revised form September 23, 2014.

Published online February 2016. 
where $\Omega=\mathbb{R}^{N}(N=2,3)$ or $\Omega \subset \mathbb{R}^{N}$ is a smooth bounded domain, $i$ is the imaginary unit, $\mu_{1}, \mu_{2}>0$ and $\beta \neq 0$ is a coupling constant. System (1.1) arises in mathematical models from several physical phenomena, especially in nonlinear optics. Physically, the solution $\Phi_{j}$ denotes the $j^{\text {th }}$ component of the beam in Kerr-like photorefractive media ( $c f .[1])$. The positive constant $\mu_{j}$ is for self-focusing in the $j^{\text {th }}$ component of the beam, and the coupling constant $\beta$ is the interaction between the two components of the beam. Problem (1.1) also arises in the Hartree-Fock theory for a double condensate, i.e., a binary mixture of Bose-Einstein condensates in two different hyperfine states $|1\rangle$ and $|2\rangle\left(c f\right.$. [14]). Physically, $\Phi_{j}$ are the corresponding condensate amplitudes, $\mu_{j}$ and $\beta$ are the intraspecies and interspecies scattering lengths. Precisely, the sign of $\mu_{j}$ represents the self-interactions of the single state $|j\rangle$. If $\mu_{j}>0$ as considered here, it is called the focusing case, in opposition to the defocusing case where $\mu_{j}<0$. Besides, the sign of $\beta$ determines whether the interactions of states $|1\rangle$ and $|2\rangle$ are repulsive or attractive, i.e., the interaction is attractive if $\beta>0$, and the interaction is repulsive if $\beta<0$, where the two states are in strong competition when $\beta$ is negative and very large.

To obtain solitary wave solutions of system (1.1), we set $\Phi_{j}(x, t)=e^{i \lambda_{j} t} u_{j}(x)$ for $j=1,2$, where $u_{j}(x)$ are real-valued functions. Then system (1.1) is reduced to the following elliptic system

$$
\begin{cases}-\Delta u_{1}+\lambda_{1} u_{1}=\mu_{1} u_{1}^{3}+\beta u_{1} u_{2}^{2} & x \in \Omega \\ -\Delta u_{2}+\lambda_{2} u_{2}=\mu_{2} u_{2}^{3}+\beta u_{1}^{2} u_{2} & x \in \Omega \\ u_{1}=u_{2}=0 & \text { on } \partial \Omega .\end{cases}
$$

Here, for the case $\Omega=\mathbb{R}^{N}$, the boundary condition $u_{1}=u_{2}=0$ on $\partial \Omega$ means

$$
u_{1}(x), u_{2}(x) \rightarrow 0 \quad \text { as }|x| \rightarrow+\infty .
$$

It is well known that finite energy solutions of (1.2) correspond to the critical points of $C^{2}$ functional $E_{\beta}: H_{0}^{1}(\Omega) \times H_{0}^{1}(\Omega) \rightarrow \mathbb{R}$ given by

$$
\begin{aligned}
E_{\beta}\left(u_{1}, u_{2}\right):= & \frac{1}{2} \int_{\Omega}\left(\left|\nabla u_{1}\right|^{2}+\lambda_{1} u_{1}^{2}+\left|\nabla u_{2}\right|^{2}+\lambda_{2} u_{2}^{2}\right) d x \\
& -\frac{1}{4} \int_{\Omega}\left(\mu_{1} u_{1}^{4}+\mu_{2} u_{2}^{4}\right) d x-\frac{\beta}{2} \int_{\Omega} u_{1}^{2} u_{2}^{2} d x .
\end{aligned}
$$

Definition 1.1. We call a solution $\left(u_{1}, u_{2}\right)$ nontrivial if $u_{j} \not \equiv 0$ for $j=1,2$, a solution $\left(u_{1}, u_{2}\right)$ semi-trivial if $\left(u_{1}, u_{2}\right)$ is type of $\left(u_{1}, 0\right)$ or $\left(0, u_{2}\right)$. A solution $\left(u_{1}, u_{2}\right)$ is called positive if $u_{j}>0$ in $\Omega$ for $j=1,2$, a solution $\left(u_{1}, u_{2}\right)$ sign-changing if both $u_{1}$ and $u_{2}$ change sign, a solution $\left(u_{1}, u_{2}\right)$ semi-nodal if one component is positive and the other one changes sign.

Definition 1.2. A nontrivial solution $\left(u_{1}, u_{2}\right)$ is called a least energy solution, if it has the least energy among all nontrivial solutions, i.e., $E_{\beta}\left(u_{1}, u_{2}\right) \leq E_{\beta}\left(v_{1}, v_{2}\right)$ for any nontrivial solution $\left(v_{1}, v_{2}\right)$ of (1.2). A sign-changing solution $\left(u_{1}, u_{2}\right)$ is called a least energy sign-changing solution, if it has the least energy among all sign-changing solutions. 
In the last decades, system (1.2) has received great interest from many mathematicians. When $\Omega$ is the entire space $\mathbb{R}^{N}$, the existence of least energy and other finite energy solutions of (1.2) was studied in $[2,5,6,10,16,19-22,27,31]$ and references therein. In particular, when $\beta>0$ is sufficiently large, multiple radially symmetric sign-changing solutions of (1.2) were obtained in [22], where the radial symmetry of $\mathbb{R}^{N}$ plays a crucial role in their proof. Under assumptions $\lambda_{i}>0, \mu_{i}>0$ and $\beta<0$, Liu and Wang [20] proved that system (1.2) has infinitely many nontrivial solutions. In fact, they studied a general $m$-coupled system $(m \geq 2)$. Remark that whether solutions obtained in [20] are positive or sign-changing is not known.

When $\Omega \subset \mathbb{R}^{N}(N=2,3)$ is a smooth bounded domain, there are also many papers studying (1.2). Lin and Wei [17] proved that a least energy solution of (1.2) exists within the range $\beta \in\left(-\infty, \beta_{0}\right)$, where $0<\beta_{0}<\sqrt{\mu_{1} \mu_{2}}$. In case where $\lambda_{1}=\lambda_{2}>0, \mu_{1}=\mu_{2}>0$ and $\beta \leq-\mu_{1}$, Dancer, Wei and Weth [13] proved the existence of infinitely many positive solutions of (1.2), while the same result was proved for the case $\lambda_{1}=\lambda_{2}<0$ by Noris and Ramos [24]. When $\Omega$ is a ball, an interesting multiplicity result on positive radially symmetric solutions was given in [32]. Remark that, since $\lambda_{1}=\lambda_{2}$ and $\mu_{1}=\mu_{2}$, so system (1.2) is invariant under the transformation $\left(u_{1}, u_{2}\right) \mapsto\left(u_{2}, u_{1}\right)$, which plays a crucial role in [13,24,32]. Later, by using a global bifurcation approach, the result of [32] was reproved by [3] without requiring the symmetric condition $\mu_{1}=\mu_{2}$, but in their proof the assumption $\lambda_{1}=\lambda_{2}$ plays a crucial role. Under assumptions $\lambda_{i}>0, \mu_{i}>0$ and $\beta<0$ without requiring $\lambda_{1}=\lambda_{2}$ or $\mu_{1}=\mu_{2}$, Sato and Wang [28] proved that system (1.2) has infinitely many semi-positive solutions (i.e., at least one component is positive). Note that all the papers mentioned above deal with the subcritical case $N \leq 3$ (i.e., the cubic nonlinearities are all of subcritical growth). Recently, Chen and Zou [9] studied the existence and properties of least energy solutions of (1.2) in the critical case $N=4$.

In a word, for $N=2,3$, a natural question, which seems to be still open for both the entire space case and the bounded domain case, is whether (1.2) has infinitely many sign-changing solutions when $\beta<0$. This is expected by many experts but no proof has yet been obtained. Here we can give a positive answer to this open question. Since the results in the entire space case are slightly different from those in the bounded domain case, in this section we only state our results in the bounded domain case for the sake of brevity. The results in the entire space case will be given in Section 6. Our first result is as follows.

Theorem 1.3. Let $N=2,3, \Omega \subset \mathbb{R}^{N}$ is a smooth bounded domain, $\lambda_{1}, \lambda_{2}$, $\mu_{1}, \mu_{2}>0$ and $\beta<0$. Then (1.2) has infinitely many sign-changing solutions $\left(u_{n, 1}, u_{n, 2}\right)$ such that

$$
\left\|u_{n, 1}\right\|_{L^{\infty}(\Omega)}+\left\|u_{n, 2}\right\|_{L^{\infty}(\Omega)} \rightarrow+\infty \quad \text { as } n \rightarrow+\infty
$$

Remark 1.4. Comparing with $[3,13,24,32]$ where infinitely many positive solutions were obtained, we do not need any symmetric assumptions $\lambda_{1}=\lambda_{2}$ or $\mu_{1}=\mu_{2}$. 
Remark 1.5. All the papers mentioned above and this paper deal with the focusing case $\mu_{1}, \mu_{2}>0$. For the defocusing case $\mu_{1}, \mu_{2}<0$, related results can be seen in $[8,25,26,29,30]$. In particular, Tavares and Terracini [29] studied the following general $m$-coupled system

$$
\left\{\begin{array}{l}
-\Delta u_{j}-\mu_{j} u_{j}^{3}-\beta u_{j} \sum_{i \neq j} u_{i}^{2}=\lambda_{j, \beta} u_{j} \\
u_{j} \in H_{0}^{1}(\Omega) \quad j=1, \ldots, m,
\end{array}\right.
$$

where $\Omega$ is a smooth bounded domain, $\beta<0$ and $\mu_{j} \leq 0$. Then [29, Theorem 1.1] says that for each fixed $\beta<0$ and $\mu_{1}, \ldots, \mu_{m} \leq 0$, there exist infinitely many $\lambda=\left(\lambda_{1, \beta}, \ldots, \lambda_{m, \beta}\right) \in \mathbb{R}^{m}$ and $u=\left(u_{1}, \ldots, u_{m}\right) \in H_{0}^{1}\left(\Omega, \mathbb{R}^{m}\right)$ such that $(u, \lambda)$ are sign-changing solutions of (1.4). That is, for each fixed $\beta<0$ and $\mu_{1}, \ldots, \mu_{m} \leq 0, \lambda_{j, \beta}$ is not fixed a priori and appears as a Lagrange multiplier in [29]. Our result is different from [29, Theorem 1.1] on two aspects: one is that we deal with the focusing case $\mu_{j}>0$, the other one is that $\lambda_{j}, \mu_{j}$ and $\beta$ are all fixed constants in Theorem 1.3. To the best of our knowledge, our result for system (1.2) is new.

As pointed out before, Lin and Wei [17] proved for $\beta \in\left(-\infty, \beta_{0}\right)$ that (1.2) has a least energy solution which turns out to be a positive solution. Since (1.2) has infinitely many sign-changing solutions for any $\beta<0$, another natural question is whether (1.2) has a least energy sign-changing solution, which has not been studied before. Here we can prove the following result.

Theorem 1.6. Let assumptions in Theorem 1.3 hold. Then (1.2) has a least energy sign-changing solution $\left(u_{1}, u_{2}\right)$. Moreover, both $u_{1}$ and $u_{2}$ have exactly two nodal domains.

Theorems 1.3 and 1.6 are both concerned with sign-changing solutions. Besides positive solutions (see $[3,13,32]$ ) and sign-changing solutions, as defined in Definition 1.1, it is natural to suspect that (1.2) may have semi-nodal solutions. Here we can prove the following result.

Theorem 1.7. Let assumptions in Theorem 1.3 hold. Then (1.2) has infinitely many semi-nodal solutions $\left\{\left(u_{n, 1}, u_{n, 2}\right)\right\}_{n \geq 2}$ such that

(1) $u_{n, 1}$ changes sign and $u_{n, 2}$ is positive;

(2) $\left\|u_{n, 1}\right\|_{L^{\infty}(\Omega)}+\left\|u_{n, 2}\right\|_{L^{\infty}(\Omega)} \rightarrow+\infty$ as $n \rightarrow+\infty$;

(3) $u_{n, 1}$ has at most $n$ nodal domains. In particular, $u_{2,1}$ has exactly two nodal domains, and $\left(u_{2,1}, u_{2,2}\right)$ has the least energy among all nontrivial solutions whose first component changes sign.

Remark 1.8. Recently, we found that [28, Theorem 0.1] proved that (1.2) has infinitely many semi-nodal solutions for any $\beta \in\left(-\sqrt{\mu_{1} \mu_{2}}, 0\right)$. Theorem $1.7 \mathrm{im}$ proves [28, Theorem 0.1$]$ on two aspects: one is that we can obtain infinitely many semi-nodal solutions for $\beta \leq-\sqrt{\mu_{1} \mu_{2}}$; the other one is that, in [28] no properties of the form (3) can be obtained by their approach. Our proofs in this paper are completely different from [28]. 
Remark 1.9. Similarly, we can prove that (1.2) has infinitely many semi-nodal solutions $\left\{\left(v_{n, 1}, v_{n, 2}\right)\right\}_{n \geq 2}$ such that $v_{n, 1}$ is positive, $v_{n, 2}$ changes sign and has at most $n$ nodal domains. In the symmetric case where $\lambda_{1}=\lambda_{2}$ and $\mu_{1}=\mu_{2},\left(u_{n, 1}, u_{n, 2}\right)$ obtained in Theorem 1.7 and $\left(v_{n, 1}, v_{n, 2}\right)$ may be the same solution in the sense of $u_{n, 1}=v_{n, 2}$ and $u_{n, 2}=v_{n, 1}$. However, if either $\lambda_{1} \neq \lambda_{2}$ or $\mu_{1} \neq \mu_{2}$, then $\left(u_{n, 1}, u_{n, 2}\right)$ and $\left(v_{n, 1}, v_{n, 2}\right)$ are really different solutions.

We give some notations here. Throughout this paper, we denote the norm of $L^{p}(\Omega)$ by $|u|_{p}=\left(\int_{\Omega}|u|^{p} d x\right)^{\frac{1}{p}}$, the norm of $H_{0}^{1}(\Omega)$ by $\|u\|^{2}=\int_{\Omega}\left(|\nabla u|^{2}+u^{2}\right) d x$ and positive constants (possibly different in different places) by $C, C_{0}, C_{1}, \ldots$ Denote $\|u\|_{\lambda_{i}}^{2}:=\int_{\Omega}\left(|\nabla u|^{2}+\lambda_{i} u^{2}\right) d x$ for convenience. Since we assume $\lambda_{1}, \lambda_{2}>0$ here, $\|\cdot\|_{\lambda_{i}}$ are equivalent norms to $\|\cdot\|$. Define $H:=H_{0}^{1}(\Omega) \times H_{0}^{1}(\Omega)$ with norm $\left\|\left(u_{1}, u_{2}\right)\right\|_{H}^{2}:=\left\|u_{1}\right\|_{\lambda_{1}}^{2}+\left\|u_{2}\right\|_{\lambda_{2}}^{2}$.

The rest of this paper is organized as follows. In Section 2 we give the proof of Theorem 1.3. The main idea of this proof is inspired by [29], where a new notion of vector genus introduced by [29] will be used to define appropriate minimax values. Some arguments in our proof are borrowed from [29] with modifications. Remark that the ideas in [29] can not be used directly, and here we will give some new ideas. For example, to obtain nontrivial solutions of (1.2), the crucial idea in this paper is turning to study a new problem with two constraints. Somewhat surprisingly, up to our knowledge, this natural idea has never been used for (1.2) in the literature; see Remark 2.3 below. In Section 3 we will use general Nehari type manifolds to prove Theorem 1.6. By giving some modifications to arguments in Sections 2 and 3, we will prove Theorem 1.7 in Section 4.

In Section 5, we will study the limit behavior of solutions obtained here as $\beta \rightarrow-\infty$ by applying results in [26] directly. It turns out that components of the limiting profile tend to separate in different regions. This phenomenon, called phase separation, has been well studied for $L^{\infty}$-bounded positive solutions of (1.2) in the case $N=2,3$ by $[26,32,33]$. For other kinds of elliptic systems with strong competition, phase separation has also been well studied, we refer to $[7,12]$ and references therein. The main result of Section 5 is Theorem 5.1.

Finally in Section 6, we will introduce existence results of infinitely many radially symmetric sign-changing and semi-nodal solutions in the entire space case. The main results are Theorems 6.1 and 6.2. The main ideas of the proof are the same as those in Sections 2-4. However, we will see that some ideas and arguments are quite different from those in the bounded domain case.

After this paper was submitted, we learned from Z.-Q. Wang of a recent work [18], where infinitely many sign-changing solutions of the general $m$-coupled system $(m \geq 2)$ were obtained via a quite different method. We should point out that our approach here also works for the general $m$-coupled system $(m \geq 2)$.

ACKNOWLEDGEMENTS. The authors wish to thank the anonymous referee very much for careful reading and valuable comments. 


\section{Proof of Theorem 1.3}

In the sequel we assume that assumptions in Theorem 1.3 hold. Since we are only concerned with nontrivial solutions, we denote $\widetilde{H}:=\left\{\left(u_{1}, u_{2}\right) \in H: u_{i} \neq\right.$ 0 for $i=1,2\}$, which is an open subset of $H$. Write $\vec{u}=\left(u_{1}, u_{2}\right)$ for convenience.

Lemma 2.1. For any $\left(u_{1}, u_{2}\right) \in \widetilde{H}$, if

$$
|\beta|^{2}\left(\int_{\Omega} u_{1}^{2} u_{2}^{2}\right)^{2} \geq \mu_{1} \mu_{2}\left|u_{1}\right|_{4}^{4}\left|u_{2}\right|_{4}^{4},
$$

then

$$
\sup _{t_{1}, t_{2} \geq 0} E_{\beta}\left(\sqrt{t_{1}} u_{1}, \sqrt{t_{2}} u_{2}\right)=+\infty
$$

Proof. By (2.1) there exists $\alpha>0$ such that

$$
\alpha|\beta| \int_{\Omega} u_{1}^{2} u_{2}^{2} \geq \mu_{1}\left|u_{1}\right|_{4}^{4}, \quad \frac{1}{\alpha}|\beta| \int_{\Omega} u_{1}^{2} u_{2}^{2} \geq \mu_{2}\left|u_{2}\right|_{4}^{4},
$$

which implies

$$
\begin{aligned}
E_{\beta}\left(\sqrt{t} u_{1}, \sqrt{\alpha t} u_{2}\right)= & \frac{1}{2} t\left\|u_{1}\right\|_{\lambda_{1}}^{2}+\frac{1}{2} \alpha t\left\|u_{2}\right\|_{\lambda_{2}}^{2} \\
& -\frac{1}{4}\left(t^{2} \mu_{1}\left|u_{1}\right|_{4}^{4}+\alpha^{2} t^{2} \mu_{2}\left|u_{2}\right|_{4}^{4}\right)+\frac{1}{2} \alpha t^{2}|\beta| \int_{\Omega} u_{1}^{2} u_{2}^{2} \\
\geq & \frac{1}{2} t\left\|u_{1}\right\|_{\lambda_{1}}^{2}+\frac{1}{2} \alpha t\left\|u_{2}\right\|_{\lambda_{2}}^{2} \rightarrow+\infty \quad \text { as } t \rightarrow+\infty .
\end{aligned}
$$

This completes the proof.

Lemma 2.2. For any $\vec{u}=\left(u_{1}, u_{2}\right) \in \widetilde{H}$, if

$$
|\beta|^{2}\left(\int_{\Omega} u_{1}^{2} u_{2}^{2}\right)^{2}<\mu_{1} \mu_{2}\left|u_{1}\right|_{4}^{4}\left|u_{2}\right|_{4}^{4},
$$

then system

$$
\left\{\begin{array}{l}
\left\|u_{1}\right\|_{\lambda_{1}}^{2}=t_{1} \mu_{1}\left|u_{1}\right|_{4}^{4}-t_{2}|\beta| \int_{\Omega} u_{1}^{2} u_{2}^{2} \\
\left\|u_{2}\right\|_{\lambda_{2}}^{2}=t_{2} \mu_{2}\left|u_{2}\right|_{4}^{4}-t_{1}|\beta| \int_{\Omega} u_{1}^{2} u_{2}^{2}
\end{array}\right.
$$

has a unique solution

$$
\left\{\begin{array}{l}
t_{1}(\vec{u})=\frac{\mu_{2}\left|u_{2}\right|_{4}^{4}\left\|u_{1}\right\|_{\lambda_{1}}^{2}+|\beta|\left\|u_{2}\right\|_{\lambda_{2}}^{2} \int_{\Omega} u_{1}^{2} u_{2}^{2}}{\mu_{1} \mu_{2}\left|u_{1}\right|_{4}^{4}\left|u_{2}\right|_{4}^{4}-|\beta|^{2}\left(\int_{\Omega} u_{1}^{2} u_{2}^{2}\right)^{2}}>0 \\
t_{2}(\vec{u})=\frac{\mu_{1}\left|u_{1}\right|_{4}^{4}\left\|u_{2}\right\|_{\lambda_{2}}^{2}+|\beta|\left\|u_{1}\right\|_{\lambda_{1}}^{2} \int_{\Omega} u_{1}^{2} u_{2}^{2}}{\mu_{1} \mu_{2}\left|u_{1}\right|_{4}^{4}\left|u_{2}\right|_{4}^{4}-|\beta|^{2}\left(\int_{\Omega} u_{1}^{2} u_{2}^{2}\right)^{2}}>0 .
\end{array}\right.
$$


Moreover,

$$
\begin{aligned}
\sup _{t_{1}, t_{2} \geq 0} & E_{\beta}\left(\sqrt{t_{1}} u_{1}, \sqrt{t_{2}} u_{2}\right)=E_{\beta}\left(\sqrt{t_{1}(\vec{u})} u_{1}, \sqrt{t_{2}(\vec{u})} u_{2}\right) \\
= & \frac{1}{4}\left(t_{1}(\vec{u})\left\|u_{1}\right\|_{\lambda_{1}}^{2}+t_{2}(\vec{u})\left\|u_{2}\right\|_{\lambda_{2}}^{2}\right) \\
= & \frac{1}{4} \frac{\mu_{2}\left|u_{2}\right|_{4}^{4}\left\|u_{1}\right\|_{\lambda_{1}}^{4}+2|\beta|\left\|u_{1}\right\|_{\lambda_{1}}^{2}\left\|u_{2}\right\|_{\lambda_{2}}^{2} \int_{\Omega} u_{1}^{2} u_{2}^{2}+\mu_{1}\left|u_{1}\right|_{4}^{4}\left\|u_{2}\right\|_{\lambda_{2}}^{4}}{\mu_{1} \mu_{2}\left|u_{1}\right|_{4}^{4}\left|u_{2}\right|_{4}^{4}-|\beta|^{2}\left(\int_{\Omega} u_{1}^{2} u_{2}^{2}\right)^{2}}
\end{aligned}
$$

and $\left(t_{1}(\vec{u}), t_{2}(\vec{u})\right)$ is the unique maximum point of $E_{\beta}\left(\sqrt{t_{1}} u_{1}, \sqrt{t_{2}} u_{2}\right)$.

Proof. It suffices to prove (2.5). Recall that $\left(t_{1}(\vec{u}), t_{2}(\vec{u})\right)$ is the solution of $(2.3)$, we deduce that

$$
\begin{aligned}
2 t_{1} t_{2}|\beta| \int_{\Omega} u_{1}^{2} u_{2}^{2} & \leq t_{1}^{2} \frac{t_{2}(\vec{u})}{t_{1}(\vec{u})}|\beta| \int_{\Omega} u_{1}^{2} u_{2}^{2}+t_{2}^{2} \frac{t_{1}(\vec{u})}{t_{2}(\vec{u})}|\beta| \int_{\Omega} u_{1}^{2} u_{2}^{2} \\
& =t_{1}^{2} \mu_{1}\left|u_{1}\right|_{4}^{4}+t_{2}^{2} \mu_{2}\left|u_{2}\right|_{4}^{4}-\frac{t_{1}^{2}}{t_{1}(\vec{u})}\left\|u_{1}\right\|_{\lambda_{1}}^{2}-\frac{t_{2}^{2}}{t_{2}(\vec{u})}\left\|u_{2}\right\|_{\lambda_{2}}^{2} .
\end{aligned}
$$

Hence for any $t_{1}, t_{2} \geq 0$,

$$
\begin{aligned}
E_{\beta}\left(\sqrt{t_{1}} u_{1}, \sqrt{t_{2}} u_{2}\right)= & \frac{1}{2} t_{1}\left\|u_{1}\right\|_{\lambda_{1}}^{2}+\frac{1}{2} t_{2}\left\|u_{2}\right\|_{\lambda_{2}}^{2} \\
& -\frac{1}{4}\left(t_{1}^{2} \mu_{1}\left|u_{1}\right|_{4}^{4}+t_{2}^{2} \mu_{2}\left|u_{2}\right|_{4}^{4}\right)+\frac{1}{2} t_{1} t_{2}|\beta| \int_{\Omega} u_{1}^{2} u_{2}^{2} \\
\leq & \left(\frac{t_{1}}{2}-\frac{t_{1}^{2}}{4 t_{1}(\vec{u})}\right)\left\|u_{1}\right\|_{\lambda_{1}}^{2}+\left(\frac{t_{2}}{2}-\frac{t_{2}^{2}}{4 t_{2}(\vec{u})}\right)\left\|u_{2}\right\|_{\lambda_{2}}^{2} \\
\leq & \frac{1}{4}\left(t_{1}(\vec{u})\left\|u_{1}\right\|_{\lambda_{1}}^{2}+t_{2}(\vec{u})\left\|u_{2}\right\|_{\lambda_{2}}^{2}\right) \\
= & E_{\beta}\left(\sqrt{t_{1}(\vec{u})} u_{1}, \sqrt{t_{2}(\vec{u})} u_{2}\right) .
\end{aligned}
$$

Therefore $(2.5)$ holds and $\left(t_{1}(\vec{u}), t_{2}(\vec{u})\right)$ is also the unique maximum point of $E_{\beta}\left(\sqrt{t_{1}} u_{1}, \sqrt{t_{2}} u_{2}\right)$ in $[0,+\infty)^{2}$.

Define

$$
\begin{aligned}
\mathcal{M}^{*} & :=\left\{\vec{u} \in H:\left|u_{1}\right|_{4}>1 / 2,\left|u_{2}\right|_{4}>1 / 2\right\} ; \\
\mathcal{M}_{\beta}^{*} & :=\left\{\vec{u} \in \mathcal{M}^{*}: \vec{u} \text { satisfies }(2.2)\right\} ; \\
\mathcal{M}_{\beta}^{* *} & :=\left\{\vec{u} \in \mathcal{M}^{*}: \mu_{1} \mu_{2}-|\beta|^{2}\left(\int_{\Omega} u_{1}^{2} u_{2}^{2}\right)^{2}>0\right\} ; \\
\mathcal{M} & :=\left\{\vec{u} \in H:\left|u_{1}\right|_{4}=1,\left|u_{2}\right|_{4}=1\right\}, \quad \mathcal{M}_{\beta}:=\mathcal{M} \cap \mathcal{M}_{\beta}^{*} .
\end{aligned}
$$


Then $\mathcal{M}_{\beta}=\mathcal{M} \cap \mathcal{M}_{\beta}^{* *}$. By taking $\varphi_{i} \in C_{0}^{\infty}(\Omega)$ such that $\left|\varphi_{i}\right|_{4}=1$ for $i=1,2$ and $\operatorname{supp}\left(\varphi_{1}\right) \cap \operatorname{supp}\left(\varphi_{2}\right)=\emptyset$, we have $\left(\varphi_{1}, \varphi_{2}\right) \in \mathcal{M}_{\beta}$, namely $\mathcal{M}_{\beta} \neq \emptyset$. It is easy to check that $\mathcal{M}^{*}, \mathcal{M}_{\beta}^{*}, \mathcal{M}_{\beta}^{* *}$ are all open subsets of $H$ and $\mathcal{M}$ is closed. Define a new functional $J_{\beta}: \mathcal{M}^{*} \rightarrow(0,+\infty]$ by

$$
J_{\beta}(\vec{u}):= \begin{cases}\frac{1}{4} \frac{\mu_{2}\left\|u_{1}\right\|_{\lambda_{1}}^{4}+2|\beta|\left\|u_{1}\right\|_{\lambda_{1}}^{2}\left\|u_{2}\right\|_{\lambda_{2}}^{2} \int_{\Omega} u_{1}^{2} u_{2}^{2}+\mu_{1}\left\|u_{2}\right\|_{\lambda_{2}}^{4}}{\mu_{1} \mu_{2}-|\beta|^{2}\left(\int_{\Omega} u_{1}^{2} u_{2}^{2}\right)^{2}} & \text { if } \vec{u} \in \mathcal{M}_{\beta}^{* *}, \\ +\infty & \text { if } \vec{u} \in \mathcal{M}^{*} \backslash \mathcal{M}_{\beta}^{* *} .\end{cases}
$$

By the Sobolev inequality

$$
\|u\|_{\lambda_{i}}^{2} \geq C|u|_{4}^{2}, \quad \forall u \in H_{0}^{1}(\Omega), \quad i=1,2,
$$

where $C$ is a positive constant, it is easy to check that $J_{\beta}$ is continuous on $\mathcal{M}^{*}$ and $\inf _{\mathcal{M}^{*}} J_{\beta} \geq C_{1}>0$ for some constant $C_{1}$ independent of $\beta<0$. Moreover, $J_{\beta} \in C^{1}\left(\mathcal{M}_{\beta}^{* *},(0,+\infty)\right)$, and since any $\vec{u} \in \mathcal{M}_{\beta}$ is an interior point of $\mathcal{M}_{\beta}^{* *}$, a direct computation and (2.4) yield that

$$
\begin{aligned}
& J_{\beta}^{\prime}(\vec{u})(\varphi, 0)=t_{1}(\vec{u}) \int_{\Omega}\left(\nabla u_{1} \nabla \varphi+\lambda_{1} u_{1} \varphi\right)+t_{1}(\vec{u}) t_{2}(\vec{u})|\beta| \int_{\Omega} u_{1} u_{2}^{2} \varphi, \\
& J_{\beta}^{\prime}(\vec{u})(0, \psi)=t_{2}(\vec{u}) \int_{\Omega}\left(\nabla u_{2} \nabla \psi+\lambda_{2} u_{2} \psi\right)+t_{1}(\vec{u}) t_{2}(\vec{u})|\beta| \int_{\Omega} u_{1}^{2} u_{2} \psi
\end{aligned}
$$

hold for any $\vec{u} \in \mathcal{M}_{\beta}$ and $\varphi, \psi \in H_{0}^{1}(\Omega)$ (remark that (2.8)-(2.9) do not hold for $\vec{u} \in \mathcal{M}_{\beta}^{* *} \backslash \mathcal{M}_{\beta}$ ). Note that Lemmas 2.1 and 2.2 yield

$$
J_{\beta}\left(u_{1}, u_{2}\right)=\sup _{t_{1}, t_{2} \geq 0} E_{\beta}\left(\sqrt{t_{1}} u_{1}, \sqrt{t_{2}} u_{2}\right), \quad \forall\left(u_{1}, u_{2}\right) \in \mathcal{M} .
$$

To obtain nontrivial solutions of (1.2), we turn to study the new functional $J_{\beta}$ restricted to $\mathcal{M}_{\beta}$, which is a problem with two constraints.

Remark 2.3. To obtain nontrivial solutions of (1.2), in many papers (see $[9,13,16$, $17,27,32]$ for example), people usually turn to study nontrivial critical points of $E_{\beta}$ under the following Nehari manifold type constraint

$$
\left\{\left(u_{1}, u_{2}\right) \in \widetilde{H}: E_{\beta}^{\prime}\left(u_{1}, u_{2}\right)\left(u_{1}, 0\right)=E_{\beta}^{\prime}\left(u_{1}, u_{2}\right)\left(0, u_{2}\right)=0\right\},
$$

which is actually a natural constraint for any $\beta<\sqrt{\mu_{1} \mu_{2}}$ (see [27, Proposition 1.1] for example). To the best of our knowledge, our natural idea (i.e., to obtain nontrivial solutions of (1.2) by studying $J_{\beta} \mid \mathcal{M}_{\beta}$ ), has never been introduced for (1.2) in the literature.

In the following, we always let $(i, j)=(1,2)$ or $(i, j)=(2,1)$. Recall that $t_{i}(\vec{u})$ is well defined for $\vec{u} \in \mathcal{M}_{\beta}^{*}$. For any $\vec{u}=\left(u_{1}, u_{2}\right) \in \mathcal{M}_{\beta}^{*}$, let $\tilde{w}_{i} \in H_{0}^{1}(\Omega)$ be the unique solution of the following linear problem

$$
-\Delta \tilde{w}_{i}+\lambda_{i} \tilde{w}_{i}+|\beta| t_{j}(\vec{u}) u_{j}^{2} \tilde{w}_{i}=\mu_{i} t_{i}(\vec{u}) u_{i}^{3}, \quad \tilde{w}_{i} \in H_{0}^{1}(\Omega) .
$$


Since $\left|u_{i}\right|_{4}>1 / 2$, so $\tilde{w}_{i} \neq 0$ and

$$
\int_{\Omega} u_{i}^{3} \tilde{w}_{i}=\frac{1}{\mu_{i} t_{i}(\vec{u})}\left(\left\|\tilde{w}_{i}\right\|_{\lambda_{i}}^{2}+|\beta| t_{j}(\vec{u}) \int_{\Omega} u_{j}^{2} \tilde{w}_{i}^{2}\right)>0 .
$$

Define

$$
w_{i}=\alpha_{i} \tilde{w}_{i}, \quad \text { where } \alpha_{i}=\frac{1}{\int_{\Omega} u_{i}^{3} \tilde{w}_{i}}>0 .
$$

Then $w_{i}$ is the unique solution of the following problem

$$
\left\{\begin{array}{l}
-\Delta w_{i}+\lambda_{i} w_{i}+|\beta| t_{j}(\vec{u}) u_{j}^{2} w_{i}=\alpha_{i} \mu_{i} t_{i}(\vec{u}) u_{i}^{3}, \quad w_{i} \in H_{0}^{1}(\Omega), \\
\int_{\Omega} u_{i}^{3} w_{i} d x=1
\end{array}\right.
$$

Now we define an operator $K=\left(K_{1}, K_{2}\right): \mathcal{M}_{\beta}^{*} \rightarrow H$ by

$$
K(\vec{u})=\left(K_{1}(\vec{u}), K_{2}(\vec{u})\right):=\vec{w}=\left(w_{1}, w_{2}\right) .
$$

Define the transformations

$$
\sigma_{i}: H \rightarrow H \quad \text { by } \quad \sigma_{1}\left(u_{1}, u_{2}\right):=\left(-u_{1}, u_{2}\right), \sigma_{2}\left(u_{1}, u_{2}\right):=\left(u_{1},-u_{2}\right) \text {. }
$$

Then it is easy to check that

$$
K\left(\sigma_{i}(\vec{u})\right)=\sigma_{i}(K(\vec{u})), \quad i=1,2 .
$$

Lemma 2.4. $K \in C^{1}\left(\mathcal{M}_{\beta}^{*}, H\right)$.

Proof. It suffices to apply the Implicit Theorem to the $C^{1}$ map

$$
\begin{aligned}
& \Psi: \mathcal{M}_{\beta}^{*} \times H_{0}^{1}(\Omega) \times \mathbb{R} \rightarrow H_{0}^{1}(\Omega) \times \mathbb{R}, \quad \text { where } \\
& \Psi(\vec{u}, v, \alpha)=\left(v+\left(-\Delta+\lambda_{i}\right)^{-1}\left(|\beta| t_{j}(\vec{u}) u_{j}^{2} v-\alpha \mu_{i} t_{i}(\vec{u}) u_{i}^{3}\right), \int_{\Omega} u_{i}^{3} v-1\right) .
\end{aligned}
$$

Note that (2.13) holds if and only if $\Psi\left(\vec{u}, w_{i}, \alpha_{i}\right)=(0,0)$. By computing the derivative of $\Psi$ with respect to $(v, \alpha)$ at the point $\left(\vec{u}, w_{i}, \alpha_{i}\right)$ in the direction $(\bar{w}, \bar{\alpha})$, we obtain a map $\Phi: H_{0}^{1}(\Omega) \times \mathbb{R} \rightarrow H_{0}^{1}(\Omega) \times \mathbb{R}$ given by

$$
\begin{aligned}
\Phi(\bar{w}, \bar{\alpha}): & =D_{v, \alpha} \Psi\left(\vec{u}, w_{i}, \alpha_{i}\right)(\bar{w}, \bar{\alpha}) \\
& =\left(\bar{w}+\left(-\Delta+\lambda_{i}\right)^{-1}\left(|\beta| t_{j}(\vec{u}) u_{j}^{2} \bar{w}-\bar{\alpha} \mu_{i} t_{i}(\vec{u}) u_{i}^{3}\right), \int_{\Omega} u_{i}^{3} \bar{w} d x\right)
\end{aligned}
$$

If $\Phi(\bar{w}, \bar{\alpha})=(0,0)$, then we multiply the equation

$$
-\Delta \bar{w}+\lambda_{i} \bar{w}+|\beta| t_{j}(\vec{u}) u_{j}^{2} \bar{w}=\bar{\alpha} \mu_{i} t_{i}(\vec{u}) u_{i}^{3}
$$


by $\bar{w}$ and obtain

$$
\|\bar{w}\|_{\lambda_{i}}^{2} \leq \bar{\alpha} \mu_{i} t_{i}(\vec{u}) \int_{\Omega} u_{i}^{3} \bar{w} d x=0
$$

So $\bar{w}=0$ and then $\bar{\alpha} \mu_{i} t_{i}(\vec{u}) u_{i}^{3} \equiv 0$ in $\Omega$. Since $\mu_{i}>0, t_{i}(\vec{u})>0$ and $\left|u_{i}\right|_{4} \geq 1 / 2$, we see that $\bar{\alpha}=0$. Hence $\Phi$ is injective.

On the other hand, for any $(f, c) \in H_{0}^{1}(\Omega) \times \mathbb{R}$, let $v_{1}, v_{2} \in H_{0}^{1}(\Omega)$ be solutions of the linear problems

$$
\begin{aligned}
& -\Delta v_{1}+\lambda_{i} v_{1}+|\beta| t_{j}(\vec{u}) u_{j}^{2} v_{1}=\left(-\Delta+\lambda_{i}\right) f \\
& -\Delta v_{2}+\lambda_{i} v_{2}+|\beta| t_{j}(\vec{u}) u_{j}^{2} v_{2}=\mu_{i} t_{i}(\vec{u}) u_{i}^{3}
\end{aligned}
$$

Since $\left|u_{i}\right|_{4}>1 / 2$, so $v_{2} \neq 0$ and then $\int_{\Omega} u_{i}^{3} v_{2} d x>0$. Let $\alpha_{0}=\left(c-\int_{\Omega} u_{i}^{3} v_{1} d x\right) /$ $\int_{\Omega} u_{i}^{3} v_{2} d x$, then $\Phi\left(v_{1}+\alpha_{0} v_{2}, \alpha_{0}\right)=(f, c)$. Hence $\Phi$ is surjective, that is, $\Phi$ is a bijective map. This completes the proof.

Lemma 2.5. Assume that $\left\{\vec{u}_{n}=\left(u_{n, 1}, u_{n, 2}\right): n \geq 1\right\} \subset \mathcal{M}_{\beta}$ is bounded in $H$ and $\vec{u}_{n} \rightarrow \vec{u}=\left(u_{1}, u_{2}\right) \in \mathcal{M}_{\beta}$ weakly in $H$. Then there exists $\vec{w} \in H$ such that, up to a subsequence, $\vec{w}_{n}:=K\left(\vec{u}_{n}\right) \rightarrow \vec{w}$ strongly in $H$.

Proof. Recall the definition of $\mathcal{M}_{\beta}$ in (2.6), we deduce from (2.4) and (2.7) that there exists $C_{0}>0$ independent of $\vec{u} \in \mathcal{M}_{\beta}$ such that

$$
t_{i}(\vec{u})=\frac{\mu_{j}\left\|u_{i}\right\|_{\lambda_{i}}^{2}+|\beta|\left\|u_{j}\right\|_{\lambda_{j}}^{2} \int_{\Omega} u_{1}^{2} u_{2}^{2}}{\mu_{1} \mu_{2}-|\beta|^{2}\left(\int_{\Omega} u_{1}^{2} u_{2}^{2}\right)^{2}} \geq \frac{1}{\mu_{i}}\left\|u_{i}\right\|_{\lambda_{i}}^{2} \geq C_{0}, \quad \forall \vec{u} \in \mathcal{M}_{\beta}
$$

Since $\vec{u}_{n} \rightarrow \vec{u}=\left(u_{1}, u_{2}\right) \in \mathcal{M}_{\beta}$ weakly in $H$, so up to a subsequence, $u_{n, i} \rightarrow u_{i}$ strongly in $L^{4}(\Omega)$. Then

$$
\lim _{n \rightarrow \infty}\left(\mu_{1} \mu_{2}-|\beta|^{2}\left(\int_{\Omega} u_{n, 1}^{2} u_{n, 2}^{2}\right)^{2}\right)=\mu_{1} \mu_{2}-|\beta|^{2}\left(\int_{\Omega} u_{1}^{2} u_{2}^{2}\right)^{2}>0,
$$

where the assumption $\vec{u} \in \mathcal{M}_{\beta}$ is used. Hence we may assume that $t_{i}\left(\vec{u}_{n}\right)$ are uniformly bounded for any $n \geq 1$ and $i=1,2$, and up to a subsequence, $t_{i}\left(\vec{u}_{n}\right) \rightarrow$ $t_{i}>0$. Recall that $w_{n, i}=\alpha_{n, i} \tilde{w}_{n, i}$, where $\alpha_{n, i}$ and $\tilde{w}_{n, i}$ are seen in (2.11)-(2.12). By (2.11) we have

$$
\left\|\tilde{w}_{n, i}\right\|_{\lambda_{i}}^{2} \leq \mu_{i} t_{i}\left(\vec{u}_{n}\right) \int_{\Omega} u_{n, i}^{3} \tilde{w}_{n, i} d x \leq C\left|\tilde{w}_{n, i}\right|_{4} \leq C\left\|\tilde{w}_{n, i}\right\|_{\lambda_{i}},
$$

which implies that $\left\{\tilde{w}_{n, i}: n \geq 1\right\}$ are bounded in $H_{0}^{1}(\Omega)$. Up to a subsequence, we may assume that $\tilde{w}_{n, i} \rightarrow \tilde{w}_{i}$ weakly in $H_{0}^{1}(\Omega)$ and strongly in $L^{4}(\Omega)$. Then by 
(2.11) and Hölder inequality we get

$$
\begin{aligned}
& \int_{\Omega} \nabla \tilde{w}_{n, i} \nabla\left(\tilde{w}_{n, i}-\tilde{w}_{i}\right) d x+\lambda_{i} \int_{\Omega} \tilde{w}_{n, i}\left(\tilde{w}_{n, i}-\tilde{w}_{i}\right) d x \\
= & -|\beta| t_{j}\left(\vec{u}_{n}\right) \int_{\Omega} u_{n, j}^{2} \tilde{w}_{n, i}\left(\tilde{w}_{n, i}-\tilde{w}_{i}\right)+\mu_{i} t_{i}\left(\vec{u}_{n}\right) \int_{\Omega} u_{n, i}^{3}\left(\tilde{w}_{n, i}-\tilde{w}_{i}\right) d x \rightarrow 0
\end{aligned}
$$

as $n \rightarrow \infty$. Hence

$$
\left\|\tilde{w}_{n, i}\right\|_{\lambda_{i}}^{2}=\int_{\Omega}\left(\nabla \tilde{w}_{n, i} \nabla \tilde{w}_{i}+\lambda_{i} \tilde{w}_{n, i} \tilde{w}_{i}\right)+o(1)=\left\|\tilde{w}_{i}\right\|_{\lambda_{i}}^{2}+o(1),
$$

that is, $\tilde{w}_{n, i} \rightarrow \tilde{w}_{i}$ strongly in $H_{0}^{1}(\Omega)$. Again by (2.11) we know that $\tilde{w}_{i}$ satisfies

$$
-\Delta \tilde{w}_{i}+\lambda_{i} \tilde{w}_{i}+|\beta| t_{j} u_{j}^{2} \tilde{w}_{i}=\mu_{i} t_{i} u_{i}^{3}
$$

Since $\left|u_{i}\right|_{4}=1$, so $\tilde{w}_{i} \neq 0$ and then $\int_{\Omega} u_{i}^{3} \tilde{w}_{i} d x>0$, which implies that

$$
\lim _{n \rightarrow \infty} \alpha_{n, i}=\lim _{n \rightarrow \infty} \frac{1}{\int_{\Omega} u_{n, i}^{3} \tilde{w}_{n, i}}=\frac{1}{\int_{\Omega} u_{i}^{3} \tilde{w}_{i}}=: \alpha_{i}
$$

Therefore, $w_{n, i}=\alpha_{n, i} \tilde{w}_{n, i} \rightarrow \alpha_{i} \tilde{w}_{i}=: w_{i}$ strongly in $H_{0}^{1}(\Omega)$.

To continue our proof, we need to use vector genus introduced by [29] to define proper minimax energy levels. Recall (2.15) and (2.6), as in [29] we consider the class of sets

$$
\mathcal{F}=\left\{A \subset \mathcal{M}: A \text { is closed and } \sigma_{i}(\vec{u}) \in A \forall \vec{u} \in A, i=1,2\right\},
$$

and, for each $A \in \mathcal{F}$ and $k_{1}, k_{2} \in \mathbb{N}$, the class of functions

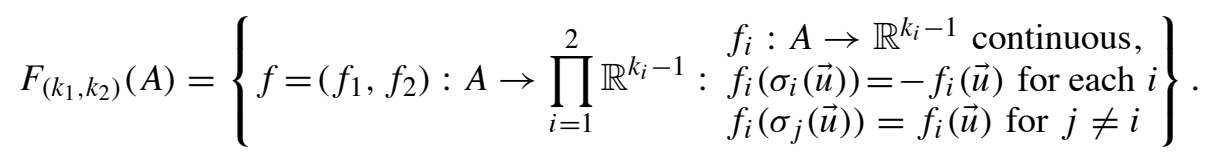

Here, we denote $\mathbb{R}^{0}:=\{0\}$. Let us recall vector genus from [29].

Definition 2.6 (Vector genus, see [29]). Let $A \in \mathcal{F}$ and take any $k_{1}, k_{2} \in \mathbb{N}$. We say that $\vec{\gamma}(A) \geq\left(k_{1}, k_{2}\right)$ if for every $f \in F_{\left(k_{1}, k_{2}\right)}(A)$ there exists $\vec{u} \in A$ such that $f(\vec{u})=\left(f_{1}(\vec{u}), f_{2}(\vec{u})\right)=(0,0)$. We denote

$$
\Gamma^{\left(k_{1}, k_{2}\right)}:=\left\{A \in \mathcal{F}: \vec{\gamma}(A) \geq\left(k_{1}, k_{2}\right)\right\} .
$$

Lemma 2.7 ([29]). With the previous notation, the following properties hold.

(i) Take $A_{1} \times A_{2} \subset \mathcal{M}$ and let $\eta_{i}: S^{k_{i}-1}:=\left\{x \in \mathbb{R}^{k_{i}}:|x|=1\right\} \rightarrow A_{i}$ be $a$ homeomorphism such that $\eta_{i}(-x)=-\eta_{i}(x)$ for every $x \in S^{k_{i}-1}, i=1,2$. Then $A_{1} \times A_{2} \in \Gamma^{\left(k_{1}, k_{2}\right)}$. 
(ii) We have $\overline{\eta(A)} \in \Gamma^{\left(k_{1}, k_{2}\right)}$ whenever $A \in \Gamma^{\left(k_{1}, k_{2}\right)}$ and a continuous map $\eta$ : $A \rightarrow \mathcal{M}$ is such that $\eta \circ \sigma_{i}=\sigma_{i} \circ \eta, \forall i=1,2$.

To obtain sign-changing solutions, as in many references such as $[4,11,34]$, we should use cones of positive functions. Precisely, we define

$$
\mathcal{P}_{i}:=\left\{\vec{u}=\left(u_{1}, u_{2}\right) \in H: u_{i} \geq 0\right\}, \quad \mathcal{P}:=\bigcup_{i=1}^{2}\left(\mathcal{P}_{i} \cup-\mathcal{P}_{i}\right) .
$$

Moreover, for $\delta>0$ we define $\mathcal{P}_{\delta}:=\left\{\vec{u} \in H: \operatorname{dist}_{4}(\vec{u}, \mathcal{P})<\delta\right\}$, where

$$
\begin{aligned}
& \operatorname{dist}_{4}(\vec{u}, \mathcal{P}):=\min \left\{\operatorname{dist}_{4}\left(u_{i}, \mathcal{P}_{i}\right), \operatorname{dist}_{4}\left(u_{i},-\mathcal{P}_{i}\right), \quad i=1,2\right\}, \\
& \operatorname{dist}_{4}\left(u_{i}, \pm \mathcal{P}_{i}\right):=\inf \left\{\left|u_{i}-v\right|_{4}: v \in \pm \mathcal{P}_{i}\right\} .
\end{aligned}
$$

Denote $u^{ \pm}:=\max \{0, \pm u\}$, then it is easy to check that $\operatorname{dist}_{4}\left(u_{i}, \pm \mathcal{P}_{i}\right)=\left|u_{i}^{\mp}\right|_{4}$.

Lemma 2.8. Let $k_{1}, k_{2} \geq 2$. Then for any $\delta<2^{-1 / 4}$ and any $A \in \Gamma^{\left(k_{1}, k_{2}\right)}$ there holds $A \backslash \mathcal{P}_{\delta} \neq \emptyset$.

Proof. Fix any $A \in \Gamma^{\left(k_{1}, k_{2}\right)}$. Consider

$$
f=\left(f_{1}, f_{2}\right): A \rightarrow \mathbb{R}^{k_{1}-1} \times \mathbb{R}^{k_{2}-1}, \quad f_{i}(\vec{u})=\left(\int_{\Omega}\left|u_{i}\right|^{3} u_{i} d x, 0, \ldots, 0\right) .
$$

Clearly $f \in F_{\left(k_{1}, k_{2}\right)}(A)$, so there exists $\vec{u} \in A$ such that $f(\vec{u})=0$. Note that $\vec{u} \in A \subset \mathcal{M}$, we conclude that

$$
\int_{\Omega}\left(u_{i}^{+}\right)^{4} d x=\int_{\Omega}\left(u_{i}^{-}\right)^{4} d x=1 / 2, \quad \text { for } i=1,2,
$$

that is, $\operatorname{dist}_{4}(\vec{u}, \mathcal{P})=2^{-1 / 4}$, and so $\vec{u} \in A \backslash \mathcal{P}_{\delta}$ for every $\delta<2^{-1 / 4}$.

Lemma 2.9. There exist $A \in \Gamma^{\left(k_{1}, k_{2}\right)}$ and a positive constant $c^{k_{1}, k_{2}} \in \mathbb{N}$ independent of $\beta<0$ such that $\sup _{A} J_{\beta} \leq c^{k_{1}, k_{2}}$ for any $\beta<0$.

Proof. Take nonempty open subsets $B_{1}, B_{2} \subset \Omega$ such that $B_{1} \cap B_{2}=\emptyset$. Let $\left\{\varphi_{k}^{i}: 1 \leq k \leq k_{i}\right\} \subset H_{0}^{1}\left(B_{i}\right)$ be linearly independent subsets, and define

$$
A_{i}:=\left\{u \in \operatorname{span}\left\{\varphi_{1}^{i}, \ldots, \varphi_{k_{i}}^{i}\right\}:|u|_{4}=1\right\} .
$$

Clearly there exists an odd homeomorphism from $S^{k_{i}-1}$ to $A_{i}$. By Lemma 2.7-(i) one has $A:=A_{1} \times A_{2} \in \Gamma^{\left(k_{1}, k_{2}\right)}$. For any $\vec{u}=\left(u_{1}, u_{2}\right) \in A$, since $u_{i} \in H_{0}^{1}\left(B_{i}\right)$, so $u_{1} \cdot u_{2} \equiv 0$, which implies $\vec{u} \in \mathcal{M}_{\beta}$ and

$$
J_{\beta}(\vec{u})=\frac{1}{4 \mu_{1} \mu_{2}}\left(\mu_{2}\left\|u_{1}\right\|_{\lambda_{1}}^{4}+\mu_{1}\left\|u_{2}\right\|_{\lambda_{2}}^{4}\right) .
$$

Since all norms of a finite dimensional linear space are equivalent, so there exists $C_{k_{i}}>0$ such that $\left\|u_{i}\right\|_{\lambda_{i}} \leq C_{k_{i}}\left|u_{i}\right|_{4}=C_{k_{i}}$ for any $u_{i} \in A_{i}$. Hence there exists $c^{k_{1}, k_{2}} \in \mathbb{N}$ independent of $\beta<0$ such that $\sup _{A} J_{\beta}(\vec{u}) \leq c^{k_{1}, k_{2}}$ for any $\beta<0$. 
For every $k_{1}, k_{2} \geq 2$ and $0<\delta<2^{-1 / 4}$, we define

$$
c_{\beta, \delta}^{k_{1}, k_{2}}:=\inf _{A \in \Gamma_{\beta}^{\left(k_{1}, k_{2}\right)}} \sup _{\vec{u} \in A \backslash \mathcal{P}_{\delta}} J_{\beta}(\vec{u}),
$$

where

$$
\Gamma_{\beta}^{\left(k_{1}, k_{2}\right)}:=\left\{A \in \Gamma^{\left(k_{1}, k_{2}\right)}: \sup _{A} J_{\beta}<c^{k_{1}, k_{2}}+1\right\} .
$$

Lemma 2.9 yields $\Gamma_{\beta}^{\left(k_{1}, k_{2}\right)} \neq \emptyset$ and so $c_{\beta, \delta}^{k_{1}, k_{2}}$ is well defined. Moreover,

$$
c_{\beta, \delta}^{k_{1}, k_{2}} \leq c^{k_{1}, k_{2}} \quad \text { for every } \beta<0 \text { and } \delta>0 .
$$

Recall that $\inf _{\mathcal{M}} J_{\beta} \geq C_{1}$, so $c_{\beta, \delta}^{k_{1}, k_{2}} \geq C_{1}>0$. We will prove that $c_{\beta, \delta}^{k_{1}, k_{2}}$ is a critical value of $E_{\beta}$ provided that $\delta>0$ is sufficiently small. As we will see in Remark 2.14, we can not replace $\Gamma_{\beta}^{\left(k_{1}, k_{2}\right)}$ by $\Gamma^{\left(k_{1}, k_{2}\right)}$ in the definition of $c_{\beta, \delta}^{k_{1}, k_{2}}$.

Lemma 2.10. For any sufficiently small $\delta \in\left(0,2^{-1 / 4}\right)$, there holds

$$
\operatorname{dist}_{4}(K(\vec{u}), \mathcal{P})<\delta / 2, \quad \forall \vec{u} \in \mathcal{M}, J_{\beta}(\vec{u}) \leq c^{k_{1}, k_{2}}+1, \operatorname{dist}_{4}(\vec{u}, \mathcal{P})<\delta .
$$

Proof. Assume by contradiction that there exist $\delta_{n} \rightarrow 0$ and $\vec{u}_{n}=\left(u_{n, 1}, u_{n, 2}\right) \in$ $\mathcal{M}$ such that $J_{\beta}\left(\vec{u}_{n}\right) \leq c^{k_{1}, k_{2}}+1$, $\operatorname{dist}_{4}\left(\vec{u}_{n}, \mathcal{P}\right)<\delta_{n}$ and $\operatorname{dist}_{4}\left(K\left(\vec{u}_{n}\right), \mathcal{P}\right) \geq \delta_{n} / 2$. Without loss of generality we may assume that $\operatorname{dist}_{4}\left(\vec{u}_{n}, \mathcal{P}\right)=\operatorname{dist}_{4}\left(u_{n, 1}, \mathcal{P}_{1}\right)$. Recall the definition of $J_{\beta}$, we see that $\vec{u}_{n} \in \mathcal{M}_{\beta}$ and

$$
c^{k_{1}, k_{2}}+1 \geq J_{\beta}\left(\vec{u}_{n}\right) \geq \frac{1}{4} \frac{\mu_{2}\left\|u_{n, 1}\right\|_{\lambda_{1}}^{4}+\mu_{1}\left\|u_{n, 2}\right\|_{\lambda_{2}}^{4}}{\mu_{1} \mu_{2}-|\beta|^{2}\left(\int_{\Omega} u_{n, 1}^{2} u_{n, 2}^{2}\right)^{2}} .
$$

This implies that $\vec{u}_{n}$ are uniformly bounded in $H$. Up to a subsequence, we may assume that $\vec{u}_{n} \rightarrow \vec{u}=\left(u_{1}, u_{2}\right)$ weakly in $H$ and strongly in $L^{4}(\Omega) \times L^{4}(\Omega)$. Hence $\left|u_{i}\right|_{4}=1$ and $\vec{u} \in \mathcal{M}$. Moreover, since (2.7) yields $\left\|u_{n, i}\right\|_{\lambda_{i}}^{2} \geq C>0$, where $C$ is independent of $n$, so we deduce from (2.24) that

$$
\mu_{1} \mu_{2}-|\beta|^{2}\left(\int_{\Omega} u_{1}^{2} u_{2}^{2}\right)^{2}=\lim _{n \rightarrow \infty}\left(\mu_{1} \mu_{2}-|\beta|^{2}\left(\int_{\Omega} u_{n, 1}^{2} u_{n, 2}^{2}\right)^{2}\right)>0,
$$

that is, $\vec{u} \in \mathcal{M}_{\beta}$. Write $K\left(\vec{u}_{n}\right)=\vec{w}_{n}=\left(w_{n, 1}, w_{n, 2}\right)$ and $w_{n, i}=\alpha_{n, i} \tilde{w}_{n, i}$ as in the proof of Lemma 2.5. Then by the proof of Lemma 2.5, we see that $t_{i}\left(\vec{u}_{n}\right)$ and $\alpha_{n, i}$ 
are all uniformly bounded. Combining this with (2.13), we deduce that

$$
\begin{aligned}
\operatorname{dist}_{4}\left(w_{n, 1}, \mathcal{P}_{1}\right)\left|w_{n, 1}^{-}\right|_{4} & =\left|w_{n, 1}^{-}\right|_{4}^{2} \leq C \int_{\Omega}\left|\nabla w_{n, 1}^{-}\right|^{2}+\lambda_{1}\left(w_{n, 1}^{-}\right)^{2} d x \\
& \leq C \int_{\Omega}\left(\left|\nabla w_{n, 1}^{-}\right|^{2}+\lambda_{1}\left(w_{n, 1}^{-}\right)^{2}+|\beta| t_{2}\left(\vec{u}_{n}\right) u_{n, 2}^{2}\left(w_{n, 1}^{-}\right)^{2}\right) \\
& =-C \alpha_{n, 1} \mu_{1} t_{1}\left(\vec{u}_{n}\right) \int_{\Omega} u_{n, 1}^{3} w_{n, 1}^{-} d x \\
& \leq C \int_{\Omega}\left(u_{n, 1}^{-}\right)^{3} w_{n, 1}^{-} d x \leq C\left|u_{n, 1}^{-}\right|_{4}^{3}\left|w_{n, 1}^{-}\right|_{4} \\
& =C \operatorname{dist}_{4}\left(u_{n, 1}, \mathcal{P}_{1}\right)^{3}\left|w_{n, 1}^{-}\right|_{4} \leq C \delta_{n}^{3}\left|w_{n, 1}^{-}\right|_{4} .
\end{aligned}
$$

So $\operatorname{dist}_{4}\left(K\left(\vec{u}_{n}\right), \mathcal{P}\right) \leq \operatorname{dist}_{4}\left(w_{n, 1}, \mathcal{P}_{1}\right) \leq C \delta_{n}^{3}<\delta_{n} / 2$ holds for $n$ sufficiently large, which is a contradiction. This completes the proof.

Now let us define a map

$$
V: \mathcal{M}_{\beta}^{*} \rightarrow H, \quad V(\vec{u}):=\vec{u}-K(\vec{u}) .
$$

We will prove that $\left(\sqrt{t_{1}(\vec{u})} u_{1}, \sqrt{t_{2}(\vec{u})} u_{2}\right)$ is a nontrivial solution of (1.2), if $\vec{u}=$ $\left(u_{1}, u_{2}\right) \in \mathcal{M}_{\beta}$ satisfies $V(\vec{u})=0$.

Lemma 2.11. Let $\vec{u}_{n}=\left(u_{n, 1}, u_{n, 2}\right) \in \mathcal{M}_{\beta}$ be such that

$$
J_{\beta}\left(\vec{u}_{n}\right) \rightarrow c<\infty \quad \text { and } \quad V\left(\vec{u}_{n}\right) \rightarrow 0 \quad \text { strongly in } H .
$$

Then, up to a subsequence, there exists $\vec{u} \in \mathcal{M}_{\beta}$ such that $\vec{u}_{n} \rightarrow \vec{u}$ strongly in $H$ and $V(\vec{u})=0$.

Proof. Without loss of generality we may assume that $J_{\beta}\left(\vec{u}_{n}\right) \leq c+1$ for all $n \geq 1$. Then by the proof of Lemma 2.10, up to a subsequence, we may assume that $\vec{u}_{n} \rightarrow \vec{u}=\left(u_{1}, u_{2}\right) \in \mathcal{M}_{\beta}$ weakly in $H$. By Lemma 2.5 , there exists $\vec{w} \in H$ such that, up to a subsequence, $\vec{w}_{n}:=K\left(\vec{u}_{n}\right)=\left(w_{n, 1}, w_{n, 2}\right) \rightarrow \vec{w}=\left(w_{1}, w_{2}\right)$ strongly in $H$. Recall $V\left(\vec{u}_{n}\right) \rightarrow 0$, we get

$$
\begin{aligned}
\int_{\Omega} \nabla u_{n, i} \nabla\left(u_{n, i}-u_{i}\right)= & \int_{\Omega} \nabla\left(w_{n, i}-w_{i}\right) \nabla\left(u_{n, i}-u_{i}\right)+\int_{\Omega} \nabla w_{i} \nabla\left(u_{n, i}-u_{i}\right) \\
& +\int_{\Omega} \nabla\left(u_{n, i}-w_{n, i}\right) \nabla\left(u_{n, i}-u_{i}\right)=o(1) .
\end{aligned}
$$

Then similarly as (2.18) we see that $\vec{u}_{n} \rightarrow \vec{u}$ strongly in $H$. By Lemma 2.4 we have $V(\vec{u})=\lim _{n \rightarrow \infty} V\left(\vec{u}_{n}\right)=0$.

Lemma 2.12. Recall $C_{0}>0$ in (2.17). Then

$$
J_{\beta}^{\prime}(\vec{u})[V(\vec{u})] \geq C_{0}\|V(\vec{u})\|_{H}^{2}, \quad \text { for any } \vec{u} \in \mathcal{M}_{\beta} .
$$


Proof. Fix any $\vec{u}=\left(u_{1}, u_{2}\right) \in \mathcal{M}_{\beta}$, write $\vec{w}=K(\vec{u})=\left(w_{1}, w_{2}\right)$ as above, then $V(\vec{u})=\left(u_{1}-w_{1}, u_{2}-w_{2}\right)$. By $(2.13)$ we have $\int_{\Omega} u_{i}^{3}\left(u_{i}-w_{i}\right) d x=1-1=0$. Then we deduce from (2.8)-(2.9), (2.13) and (2.17) that

$$
\begin{aligned}
& J_{\beta}^{\prime}(\vec{u})[V(\vec{u})] \\
= & \sum_{i=1}^{2} t_{i}(\vec{u}) \int_{\Omega}\left(\nabla u_{i} \nabla\left(u_{i}-w_{i}\right)+\lambda_{i} u_{i}\left(u_{i}-w_{i}\right)+t_{j}(\vec{u})|\beta| u_{i}\left(u_{i}-w_{i}\right) u_{j}^{2}\right) d x \\
\geq & \sum_{i=1}^{2} t_{i}(\vec{u}) \int_{\Omega}\left(\nabla u_{i} \nabla\left(u_{i}-w_{i}\right)+\lambda_{i} u_{i}\left(u_{i}-w_{i}\right)+t_{j}(\vec{u})|\beta| w_{i}\left(u_{i}-w_{i}\right) u_{j}^{2}\right) d x \\
= & \sum_{i=1}^{2} t_{i}(\vec{u}) \int_{\Omega}\left(\nabla u_{i} \nabla\left(u_{i}-w_{i}\right)+\lambda_{i} u_{i}\left(u_{i}-w_{i}\right)-\nabla w_{i} \nabla\left(u_{i}-w_{i}\right)\right. \\
= & \sum_{i=1}^{2} t_{i}(\vec{u}) \int_{\Omega} \mid \nabla\left(u_{i}-w_{i}\left(u_{i}-w_{i}\right)+\alpha_{i} \mu_{i} t_{i}(\vec{u}) u_{i}^{3}\left(u_{i}-u_{i}-w_{i}\right)\right) d x
\end{aligned}
$$

This completes the proof.

Lemma 2.13. There exists a unique global solution $\eta=\left(\eta_{1}, \eta_{2}\right):[0, \infty) \times$ $\mathcal{M}_{\beta} \rightarrow H$ for the initial value problem

$$
\frac{d}{d t} \eta(t, \vec{u})=-V(\eta(t, \vec{u})), \quad \eta(0, \vec{u})=\vec{u} \in \mathcal{M}_{\beta} .
$$

Moreover,

(i) $\eta(t, \vec{u}) \in \mathcal{M}_{\beta}$ for any $t>0$ and $u \in \mathcal{M}_{\beta}$.

(ii) $\eta\left(t, \sigma_{i}(\vec{u})\right)=\sigma_{i}(\eta(t, \vec{u}))$ for any $t>0, u \in \mathcal{M}_{\beta}$ and $i=1,2$.

(iii) For every $\vec{u} \in \mathcal{M}_{\beta}$, the map $t \mapsto J_{\beta}(\eta(t, \vec{u}))$ is non-increasing.

(iv) There exists $\delta_{0} \in\left(0,2^{-1 / 4}\right)$ such that, for every $\delta<\delta_{0}$, there holds

$$
\eta(t, \vec{u}) \in \mathcal{P}_{\delta} \quad \text { whenever } u \in \mathcal{M}_{\beta} \cap \mathcal{P}_{\delta}, J_{\beta}(u) \leq c^{k_{1}, k_{2}}+1 \text { and } t>0 .
$$

Proof. Recalling Lemma 2.4, one has $V(\vec{u}) \in C^{1}\left(\mathcal{M}_{\beta}^{*}, H\right)$. Since $\mathcal{M}_{\beta} \subset \mathcal{M}_{\beta}^{*}$ and $\mathcal{M}_{\beta}^{*}$ is open, so (2.25) has a unique solution $\eta:\left[0, T_{\max }\right) \times \mathcal{M}_{\beta} \rightarrow H$, where $T_{\max }>0$ is the maximal time such that $\eta(t, \vec{u}) \in \mathcal{M}_{\beta}^{*}$ for all $t \in\left[0, T_{\max }\right.$ ) (note that $V(\cdot)$ is defined only on $\mathcal{M}_{\beta}^{*}$ ). We should prove $T_{\max }=+\infty$ for any $\vec{u} \in \mathcal{M}_{\beta}$. Fixed any $\vec{u}=\left(u_{1}, u_{2}\right) \in \mathcal{M}_{\beta}$, one has

$$
\begin{aligned}
\frac{d}{d t} \int_{\Omega} \eta_{i}(t, \vec{u})^{4} d x & =-4 \int_{\Omega} \eta_{i}(t, \vec{u})^{3}\left(\eta_{i}(t, \vec{u})-K_{i}(\eta(t, \vec{u}))\right) d x \\
& =4-4 \int_{\Omega} \eta_{i}(t, \vec{u})^{4} d x, \quad \forall 0<t<T_{\max },
\end{aligned}
$$


that is

$$
\frac{d}{d t}\left[e^{4 t}\left(\int_{\Omega} \eta_{i}(t, \vec{u})^{4} d x-1\right)\right]=0 .
$$

Recalling $\int_{\Omega} \eta_{i}(0, \vec{u})^{4} d x=\int_{\Omega} u_{i}^{4} d x=1$, we see that

$$
\int_{\Omega} \eta_{i}(t, \vec{u})^{4} d x \equiv 1 \quad \text { for all } 0 \leq t<T_{\max }
$$

So $\eta(t, \vec{u}) \in \mathcal{M}$, that is $\eta(t, \vec{u}) \in \mathcal{M} \cap \mathcal{M}_{\beta}^{*}=\mathcal{M}_{\beta}$ for all $t \in\left[0, T_{\max }\right)$. Assume by contradiction that $T_{\max }<+\infty$, then either $\eta\left(T_{\max }, \vec{u}\right) \in \mathcal{M} \backslash \mathcal{M}_{\beta}^{*}$ or $\lim _{t \rightarrow T_{\max }}\|\eta(t, \vec{u})\|_{H}=+\infty$. If $\eta\left(T_{\max }, \vec{u}\right) \in \mathcal{M} \backslash \mathcal{M}_{\beta}^{*}$, then the definition of $J_{\beta}$ yields $J_{\beta}\left(\eta\left(T_{\max }, \vec{u}\right)\right)=+\infty$. Since $\eta(t, \vec{u}) \in \mathcal{M}_{\beta}$ for any $t \in\left[0, T_{\max }\right)$, we deduce from Lemma 2.12 that

$$
\begin{aligned}
J_{\beta}\left(\eta\left(T_{\max }, \vec{u}\right)\right) & =J_{\beta}(\eta(0, \vec{u}))+\int_{0}^{T_{\max }} \frac{d}{d t} J_{\beta}(\eta(t, \vec{u})) d t \\
& =J_{\beta}(\vec{u})-\int_{0}^{T_{\max }} J_{\beta}^{\prime}(\eta(t, \vec{u}))[V(\eta(t, \vec{u}))] d t \\
& \leq J_{\beta}(\vec{u})-C_{0} \int_{0}^{T_{\max }}\|V(\eta(t, \vec{u}))\|_{H}^{2} d t \leq J_{\beta}(\vec{u})<+\infty,
\end{aligned}
$$

a contradiction. So $\lim _{t \rightarrow T_{\max }}\|\eta(t, \vec{u})\|_{H}=+\infty$. Similarly as (2.26), we see that $J_{\beta}(\eta(t, \vec{u})) \leq J_{\beta}(\vec{u})<+\infty$ for all $t \in\left[0, T_{\max }\right)$, and so

$$
\frac{1}{4 \mu_{1} \mu_{2}}\left(\mu_{2}\left\|\eta_{1}(t, \vec{u})\right\|_{\lambda_{1}}^{4}+\mu_{1}\left\|\eta_{2}(t, \vec{u})\right\|_{\lambda_{2}}^{4}\right) \leq J_{\beta}(\eta(t, \vec{u})) \leq J_{\beta}(\vec{u})<+\infty,
$$

which means that $\|\eta(t, \vec{u})\|_{H}^{2}$ are uniformly bounded for all $\left[0, T_{\max }\right)$, also a contradiction. Hence $T_{\max }=+\infty$ and (i), (iii) hold.

By (2.16) we have $V\left(\sigma_{i}(\vec{u})\right)=\sigma_{i}(V(\vec{u}))$. Then by the uniqueness of solutions of the initial value problem (2.25), it is easy to check that (ii) holds.

Finally, let $\delta_{0} \in\left(0,2^{-1 / 4}\right)$ such that Lemma 2.10 holds for every $\delta<\delta_{0}$. For any $\vec{u} \in \mathcal{M}_{\beta}$ with $J_{\beta}(\vec{u}) \leq c^{k_{1}, k_{2}}+1$ and $\operatorname{dist}_{4}(\vec{u}, \mathcal{P})=\delta<\delta_{0}$, since

$$
\eta(t, \vec{u})=\vec{u}+t \frac{d}{d t} \eta(0, \vec{u})+o(t)=\vec{u}-t V(\vec{u})+o(t)=(1-t) \vec{u}+t K(\vec{u})+o(t),
$$

we see from Lemma 2.10 that

$$
\begin{aligned}
\operatorname{dist}_{4}(\eta(t, \vec{u}), \mathcal{P}) & =\operatorname{dist}_{4}((1-t) \vec{u}+t K(\vec{u})+o(t), \mathcal{P}) \\
& \leq(1-t) \operatorname{dist}_{4}(\vec{u}, \mathcal{P})+t \operatorname{dist}_{4}(K(\vec{u}), \mathcal{P})+o(t) \\
& \leq(1-t) \delta+t \delta / 2+o(t)<\delta
\end{aligned}
$$

for $t>0$ sufficiently small. Hence (iv) holds. 
Now we are in a position to prove Theorem 1.3.

Proof of Theorem 1.3.

Step 1. Take any $\delta \in\left(0, \delta_{0}\right)$. We prove that (1.2) has a sign-changing solution $\left(\tilde{u}_{1}, \tilde{u}_{2}\right) \in H$ such that $E_{\beta}\left(\tilde{u}_{1}, \tilde{u}_{2}\right)=c_{\beta, \delta}^{k_{1}, k_{2}}$.

Write $c_{\beta, \delta}^{k_{1}, k_{2}}$ simply by $c$ in this step. We claim that there exists a sequence $\left\{\vec{u}_{n}: n \geq 1\right\} \subset \mathcal{M}_{\beta}$ such that

$$
J_{\beta}\left(\vec{u}_{n}\right) \rightarrow c, V\left(\vec{u}_{n}\right) \rightarrow 0 \text { as } n \rightarrow \infty, \text { and } \operatorname{dist}_{4}\left(\vec{u}_{n}, \mathcal{P}\right) \geq \delta, \quad \forall n \in \mathbb{N} .
$$

If (2.27) does not hold, there exists small $\varepsilon \in(0,1)$ such that

$$
\|V(\vec{u})\|_{H}^{2} \geq \varepsilon, \quad \forall u \in \mathcal{M}_{\beta},\left|J_{\beta}(\vec{u})-c\right| \leq 2 \varepsilon, \operatorname{dist}_{4}(\vec{u}, \mathcal{P}) \geq \delta .
$$

Recall the definition of $c$ in (2.22), there exists $A \in \Gamma_{\beta}^{\left(k_{1}, k_{2}\right)}$ such that

$$
\sup _{A \backslash \mathcal{P}_{\delta}} J_{\beta}<c+\varepsilon
$$

Since $\sup _{A} J_{\beta}<c^{k_{1}, k_{2}}+1$, so $A \subset \mathcal{M}_{\beta}$. Then we can consider $B=\eta\left(2 / C_{0}, A\right)$, where $\eta$ is in Lemma 2.13 and $C_{0}$ is in (2.17). By Lemma 2.7-(ii) and Lemma 2.13-(ii) we have $B \in \Gamma^{\left(k_{1}, k_{2}\right)}$. Again by Lemma 2.13-(iii), we have $\sup _{B} J_{\beta} \leq$ $\sup _{A} J_{\beta}<c^{k_{1}, k_{2}}+1$, that is $B \in \Gamma_{\beta}^{\left(k_{1}, k_{2}\right)}$ and so $\sup _{B \backslash \mathcal{P}_{\delta}} J_{\beta} \geq c$. Then by Lemma 2.8 we can take $\vec{u} \in A$ such that $\eta\left(2 / C_{0}, \vec{u}\right) \in B \backslash \mathcal{P}_{\delta}$ and

$$
c-\varepsilon \leq \sup _{B \backslash \mathcal{P}_{\delta}} J_{\beta}-\varepsilon<J_{\beta}\left(\eta\left(2 / C_{0}, \vec{u}\right)\right) .
$$

Since $J_{\beta}(\eta(t, \vec{u})) \leq J_{\beta}(\vec{u})<c^{k_{1}, k_{2}}+1$ for any $t>0$, Lemma 2.13-(iv) yields $\eta(t, \vec{u}) \notin \mathcal{P}_{\delta}$ for any $t \in\left[0,2 / C_{0}\right]$. In particular, $\vec{u} \notin \mathcal{P}_{\delta}$ and so $J_{\beta}(\vec{u})<c+\varepsilon$. Then for any $t \in\left[0,2 / C_{0}\right]$, we have

$$
c-\varepsilon<J_{\beta}\left(\eta\left(2 / C_{0}, \vec{u}\right)\right) \leq J_{\beta}(\eta(t, \vec{u})) \leq J_{\beta}(\vec{u})<c+\varepsilon,
$$

which implies $\|V(\eta(t, \vec{u}))\|_{H}^{2} \geq \varepsilon$ and

$$
\frac{d}{d t} J_{\beta}(\eta(t, \vec{u}))=-J_{\beta}^{\prime}(\eta(t, \vec{u}))[V(\eta(t, \vec{u}))] \leq-C_{0}\|V(\eta(t, \vec{u}))\|_{H}^{2} \leq-C_{0} \varepsilon
$$

for every $t \in\left[0,2 / C_{0}\right]$. Hence,

$$
c-\varepsilon<J_{\beta}\left(\eta\left(2 / C_{0}, \vec{u}\right)\right) \leq J_{\beta}(\vec{u})-\int_{0}^{2 / C_{0}} C_{0} \varepsilon d t<c+\varepsilon-2 \varepsilon=c-\varepsilon,
$$

a contradiction. Therefore (2.27) holds, and by Lemma 2.11, up to a subsequence, there exists $\vec{u}=\left(u_{1}, u_{2}\right) \in \mathcal{M}_{\beta}$ such that $\vec{u}_{n} \rightarrow \vec{u}$ strongly in $H$ and $V(\vec{u})=0$, 
$J_{\beta}(\vec{u})=c=c_{\beta, \delta}^{k_{1}, k_{2}}$. Since $\operatorname{dist}_{4}\left(\vec{u}_{n}, \mathcal{P}\right) \geq \delta$, so $\operatorname{dist}_{4}(\vec{u}, \mathcal{P}) \geq \delta$, which implies that both $u_{1}$ and $u_{2}$ are sign-changing.

Since $V(\vec{u})=0$, so $\vec{u}=K(\vec{u})$. Combining this with (2.13)-(2.14), we see that $\vec{u}$ satisfies

$$
\left\{\begin{array}{l}
-\Delta u_{1}+\lambda_{1} u_{1}+|\beta| t_{2}(\vec{u}) u_{2}^{2} u_{1}=\alpha_{1} \mu_{1} t_{1}(\vec{u}) u_{1}^{3}, \\
-\Delta u_{2}+\lambda_{2} u_{2}+|\beta| t_{1}(\vec{u}) u_{1}^{2} u_{2}=\alpha_{2} \mu_{2} t_{2}(\vec{u}) u_{2}^{3} .
\end{array}\right.
$$

Recall that $\left|u_{i}\right|_{4}=1$ and $t_{i}(\vec{u})$ satisfies (2.3). Multiplying (2.28) by $u_{i}$ and integrating over $\Omega$, we obtain that $\alpha_{1}=\alpha_{2}=1$. Again by (2.28), we see that $\left(\tilde{u}_{1}, \tilde{u}_{2}\right):=\left(\sqrt{t_{1}(\vec{u})} u_{1}, \sqrt{t_{2}(\vec{u})} u_{2}\right)$ is a sign-changing solution of the original problem (1.2). Moreover, (2.5) and (2.10) yield

$$
E_{\beta}\left(\tilde{u}_{1}, \tilde{u}_{2}\right)=J_{\beta}\left(u_{1}, u_{2}\right)=c_{\beta, \delta}^{k_{1}, k_{2}} .
$$

This completes the proof of Step 1.

Step 2. We prove that (1.2) has infinitely many sign-changing solutions $\left(u_{n, 1}, u_{n, 2}\right)$ such that

$$
\left\|u_{n, 1}\right\|_{L^{\infty}(\Omega)}+\left\|u_{n, 2}\right\|_{L^{\infty}(\Omega)} \rightarrow+\infty \quad \text { as } n \rightarrow+\infty .
$$

It suffices to prove that

$$
\lim _{k_{1} \rightarrow \infty} \inf _{0<\delta \leq 2^{-5 / 4}} c_{\beta, \delta}^{k_{1}, k_{2}}=+\infty
$$

Assume by contradiction that there exist $k_{1}^{n} \rightarrow \infty, \delta_{n} \in\left(0,2^{-5 / 4}\right]$ and a positive constant $C$ such that $c_{\beta, \delta_{n}}^{k_{1}^{n}, k_{2}} \leq C$ for every $n \in \mathbb{N}$. Then there exists $A_{n} \in$ $\Gamma_{\beta}^{\left(k_{1}^{n}, k_{2}\right)}$ such that

$$
\sup _{A_{n} \backslash \mathcal{P}_{\delta_{n}}} J_{\beta} \leq C+1, \quad \forall n \in \mathbb{N} .
$$

Let $\left\{\varphi_{k}\right\}_{k} \subset H_{0}^{1}(\Omega)$ be the sequence of eigenfunctions of $\left(-\Delta, H_{0}^{1}(\Omega)\right)$ associated to the eigenvalues $\left\{\Lambda_{k}\right\}_{k}$, then $\Lambda_{k} \rightarrow+\infty$ as $k \rightarrow+\infty$. Define maps $g^{n}=\left(g_{1}^{n}, g_{2}^{n}\right): A_{n} \rightarrow \mathbb{R}^{k_{1}^{n}-1} \times \mathbb{R}^{k_{2}-1}$ by

$$
\begin{aligned}
& g_{1}^{n}(\vec{u}):=\left(\int_{\Omega} \varphi_{1} u_{1}, \ldots, \int_{\Omega} \varphi_{k_{1}^{n}-2} u_{1}, \int_{\Omega}\left|u_{1}\right|^{3} u_{1}\right), \\
& g_{2}^{n}(\vec{u}):=\left(\int_{\Omega} \varphi_{1} u_{2}, \ldots, \int_{\Omega} \varphi_{k_{2}-2} u_{2}, \int_{\Omega}\left|u_{2}\right|^{3} u_{2}\right) .
\end{aligned}
$$

Then $g^{n} \in F_{\left(k_{1}^{n}, k_{2}\right)}\left(A_{n}\right)$ and so there exists $\vec{u}^{n}=\left(u_{1}^{n}, u_{2}^{n}\right) \in A_{n}$ such that $g^{n}\left(\vec{u}^{n}\right)=$ 0 . As in the proof of Lemma 2.8, firstly this means $\vec{u}^{n} \in A_{n} \backslash \mathcal{P}_{\delta_{n}}$ and so $J_{\beta}\left(\vec{u}^{n}\right) \leq$ $C+1$ for every $n \in \mathbb{N}$. Secondly, we have $u_{1}^{n} \in \operatorname{span}\left\{\varphi_{1}, \ldots, \varphi_{k_{1}^{n}-2}\right\}^{\perp}$ and so $\int_{\Omega}\left|\nabla u_{1}^{n}\right|^{2} \geq \Lambda_{k_{1}^{n}-1} \int_{\Omega}\left|u_{1}^{n}\right|^{2}$. Recall that

$$
C+1 \geq J_{\beta}\left(\vec{u}^{n}\right) \geq \frac{1}{4 \mu_{1}}\left\|u_{1}^{n}\right\|_{\lambda_{1}}^{4},
$$


we see that $u_{1}^{n}$ are uniformly bounded in $H_{0}^{1}(\Omega)$. Up to a subsequence, we may assume that $u_{1}^{n} \rightarrow u_{1}$ weakly in $H_{0}^{1}(\Omega)$ and strongly in $L^{2}(\Omega) \cap L^{4}(\Omega)$. Since

$$
\int_{\Omega}\left|u_{1}^{n}\right|^{2} \leq \frac{1}{\Lambda_{k_{1}^{n}-1}} \int_{\Omega}\left|\nabla u_{1}^{n}\right|^{2} \rightarrow 0 \quad \text { as } n \rightarrow+\infty,
$$

so $u_{1}=0$. On the other hand, $\vec{u}^{n} \in A_{n} \subset \mathcal{M}$ yields $\left|u_{1}^{n}\right|_{4}=1$ for any $n$, so $\left|u_{1}\right|_{4}=1$, a contradiction. Therefore (2.30) holds and so (1.2) has infinitely many sign-changing solutions $\left(u_{n, 1}, u_{n, 2}\right)$ such that $E_{\beta}\left(u_{n, 1}, u_{n, 2}\right) \rightarrow+\infty$ as $n \rightarrow \infty$. By standard elliptic regularity theory, we see that $u_{n, i} \in L^{\infty}(\Omega)$. Since

$$
\begin{aligned}
4 E_{\beta}\left(u_{n, 1}, u_{n, 2}\right) & =\mu_{1}\left|u_{n, 1}\right|_{4}^{4}+\mu_{2}\left|u_{n, 2}\right|_{4}^{4}-2|\beta| \int_{\Omega} u_{n, 1}^{2} u_{n, 2}^{2} \\
& \leq \mu_{1}|\Omega|\left\|u_{n, 1}\right\|_{L^{\infty}(\Omega)}^{4}+\mu_{2}|\Omega|\left\|u_{n, 2}\right\|_{L^{\infty}(\Omega)}^{4},
\end{aligned}
$$

so (2.29) holds. Here $|\Omega|$ denotes the Lebesgue measure of $\Omega$. This completes the proof.

Remark 2.14. If $A \in \Gamma^{\left(k_{1}, k_{2}\right)} \backslash \Gamma_{\beta}^{\left(k_{1}, k_{2}\right)}$, we can not consider the set $\eta\left(2 / C_{0}, A\right)$ in the proof of Theorem 1.3, because $\eta(t, \cdot)$ can not be defined on the whole $\mathcal{M}$ for any $t>0$ and so $\eta\left(2 / C_{0}, A\right)$ is not well defined. Hence we can not replace $\Gamma_{\beta}^{\left(k_{1}, k_{2}\right)}$ by $\Gamma^{\left(k_{1}, k_{2}\right)}$ in the definition of $c_{\beta, \delta}^{k_{1}, k_{2}}$. Define

$$
\widetilde{\Gamma}_{\beta}^{\left(k_{1}, k_{2}\right)}:=\left\{A \in \Gamma^{\left(k_{1}, k_{2}\right)}: \sup _{A} J_{\beta}<+\infty\right\} .
$$

Then for any $A \in \widetilde{\Gamma}_{\beta}^{\left(k_{1}, k_{2}\right)}$, the set $B=\eta\left(2 / C_{0}, A\right)$ is well defined. Take $\vec{u} \in A$ such that $\eta\left(2 / C_{0}, \vec{u}\right) \in B \backslash \mathcal{P}_{\delta}$ as in the proof of Theorem 1.3. Then, since we do not know whether $J_{\beta}(\vec{u}) \leq c^{k_{1}, k_{2}}+1$ holds or not, it seems impossible for us to prove $\vec{u} \notin \mathcal{P}_{\delta}$, which plays a crucial role in the proof of Theorem 1.3. Therefore we can not replace $\Gamma_{\beta}^{\left(k_{1}, k_{2}\right)}$ by $\widetilde{\Gamma}_{\beta}^{\left(k_{1}, k_{2}\right)}$ in the definition of $c_{\beta, \delta}^{k_{1}, k_{2}}$ either.

\section{Proof of Theorem 1.6}

In this section, let $k_{1}=k_{2}=2$ and take $\delta>0$ small enough such that $c_{\beta, \delta}^{2,2}$ is a critical value of $E_{\beta}$. Write $c_{\beta, \delta}^{2,2}$ by $c$ for simplicity. By the proof of Theorem 1.3 we see that (1.2) has a sign-changing solution $\vec{U}=\left(U_{1}, U_{2}\right)$ such that

$$
E_{\beta}(\vec{U})=c \leq c^{2,2} .
$$

We will prove that $\vec{U}$ is a least energy sign-changing solution. To do this, let us define

$$
\tilde{c}:=\inf _{\vec{u} \in \mathcal{N}_{\beta}} E_{\beta}(\vec{u})
$$


where

$$
\begin{gathered}
\mathcal{N}_{\beta}:=\left\{\vec{u}=\left(u_{1}, u_{2}\right) \in H: \text { both } u_{1} \text { and } u_{2}\right. \text { change sign, } \\
\left.E_{\beta}^{\prime}(\vec{u})\left(u_{1}^{ \pm}, 0\right)=0, \quad E_{\beta}^{\prime}(\vec{u})\left(0, u_{2}^{ \pm}\right)=0\right\} .
\end{gathered}
$$

Then any sign-changing solutions belong to $\mathcal{N}_{\beta}$. In particular, $\vec{U} \in \mathcal{N}_{\beta}$ and so $\tilde{c} \leq E_{\beta}(\vec{U})=c \leq c^{2,2}$. To prove the opposite inequality $\tilde{c} \geq c$, we need the following lemma.

Lemma 3.1. Let $\vec{u}=\left(u_{1}, u_{2}\right) \in \mathcal{N}_{\beta}$, then

$$
E_{\beta}\left(u_{1}, u_{2}\right)=\sup _{t_{1}^{ \pm}, t_{2}^{ \pm} \geq 0} E_{\beta}\left(\sqrt{t_{1}^{+}} u_{1}^{+}-\sqrt{t_{1}^{-}} u_{1}^{-}, \sqrt{t_{2}^{+}} u_{2}^{+}-\sqrt{t_{2}^{-}} u_{2}^{-}\right)
$$

Proof. Note that $E_{\beta}^{\prime}(\vec{u})\left(u_{1}^{ \pm}, 0\right)=0$ and $E_{\beta}^{\prime}(\vec{u})\left(0, u_{2}^{ \pm}\right)=0$ yield

$$
\mu_{i}\left|u_{i}^{ \pm}\right|_{4}^{4}=\left\|u_{i}^{ \pm}\right\|_{\lambda_{i}}^{2}+|\beta| \int_{\Omega}\left|u_{i}^{ \pm}\right|^{2} u_{j}^{2}, \quad i=1,2 .
$$

Then

$$
\begin{aligned}
& 2|\beta| \int_{\Omega}\left|\sqrt{t_{1}^{+}} u_{1}^{+}-\sqrt{t_{1}^{-}} u_{1}^{-}\right|^{2}\left|\sqrt{t_{2}^{+}} u_{2}^{+}-\sqrt{t_{2}^{-}} u_{2}^{-}\right|^{2} \\
= & 2|\beta| t_{1}^{+} t_{2}^{+} \int_{\Omega}\left(u_{1}^{+}\right)^{2}\left(u_{2}^{+}\right)^{2}+2|\beta| t_{1}^{+} t_{2}^{-} \int_{\Omega}\left(u_{1}^{+}\right)^{2}\left(u_{2}^{-}\right)^{2} \\
& +2|\beta| t_{1}^{-} t_{2}^{+} \int_{\Omega}\left(u_{1}^{-}\right)^{2}\left(u_{2}^{+}\right)^{2}+2|\beta| t_{1}^{-} t_{2}^{-} \int_{\Omega}\left(u_{1}^{-}\right)^{2}\left(u_{2}^{-}\right)^{2} \\
\leq & |\beta|\left[\left(t_{1}^{+}\right)^{2}+\left(t_{2}^{+}\right)^{2}\right] \int_{\Omega}\left(u_{1}^{+}\right)^{2}\left(u_{2}^{+}\right)^{2}+|\beta|\left[\left(t_{1}^{+}\right)^{2}+\left(t_{2}^{-}\right)^{2}\right] \int_{\Omega}\left(u_{1}^{+}\right)^{2}\left(u_{2}^{-}\right)^{2} \\
& +|\beta|\left[\left(t_{1}^{-}\right)^{2}+\left(t_{2}^{+}\right)^{2}\right] \int_{\Omega}\left(u_{1}^{-}\right)^{2}\left(u_{2}^{+}\right)^{2}+|\beta|\left[\left(t_{1}^{-}\right)^{2}+\left(t_{2}^{-}\right)^{2}\right] \int_{\Omega}\left(u_{1}^{-}\right)^{2}\left(u_{2}^{-}\right)^{2} \\
= & |\beta|\left(t_{1}^{+}\right)^{2} \int_{\Omega}\left(u_{1}^{+}\right)^{2} u_{2}^{2}+|\beta|\left(t_{1}^{-}\right)^{2} \int_{\Omega}\left(u_{1}^{-}\right)^{2} u_{2}^{2} \\
& +|\beta|\left(t_{2}^{+}\right)^{2} \int_{\Omega} u_{1}^{2}\left(u_{2}^{+}\right)^{2}+|\beta|\left(t_{2}^{-}\right)^{2} \int_{\Omega} u_{1}^{2}\left(u_{2}^{-}\right)^{2} \\
= & \left(t_{1}^{+}\right)^{2} \mu_{1}\left|u_{1}^{+}\right|_{4}^{4}+\left(t_{1}^{-}\right)^{2} \mu_{1}\left|u_{1}^{-}\right|_{4}^{4}+\left(t_{2}^{+}\right)^{2} \mu_{2}\left|u_{2}^{+}\right|_{4}^{4}+\left(t_{2}^{-}\right)^{2} \mu_{2}\left|u_{2}^{-}\right|_{4}^{4} \\
& -\left(t_{1}^{+}\right)^{2}\left\|u_{1}^{+}\right\|_{\lambda_{1}}^{2}-\left(t_{1}^{-}\right)^{2}\left\|u_{1}^{-}\right\|_{\lambda_{1}}^{2}-\left(t_{2}^{+}\right)^{2}\left\|u_{2}^{+}\right\|_{\lambda_{2}}^{2}-\left(t_{2}^{-}\right)^{2}\left\|u_{2}^{-}\right\|_{\lambda_{2}}^{2} .
\end{aligned}
$$


Hence for any $t_{1}^{ \pm}, t_{2}^{ \pm} \geq 0$ we deduce that

$$
\begin{aligned}
& E_{\beta}\left(\sqrt{t_{1}^{+}} u_{1}^{+}-\sqrt{t_{1}^{-}} u_{1}^{-}, \sqrt{t_{2}^{+}} u_{2}^{+}-\sqrt{t_{2}^{-}} u_{2}^{-}\right) \\
= & \frac{1}{2} t_{1}^{+}\left\|u_{1}^{+}\right\|_{\lambda_{1}}^{2}+\frac{1}{2} t_{1}^{-}\left\|u_{1}^{-}\right\|_{\lambda_{1}}^{2}+\frac{1}{2} t_{2}^{+}\left\|u_{2}^{+}\right\|_{\lambda_{2}}^{2}+\frac{1}{2} t_{2}^{-}\left\|u_{2}^{-}\right\|_{\lambda_{2}}^{2} \\
& -\frac{1}{4}\left[\left(t_{1}^{+}\right)^{2} \mu_{1}\left|u_{1}^{+}\right|_{4}^{4}+\left(t_{1}^{-}\right)^{2} \mu_{1}\left|u_{1}^{-}\right|_{4}^{4}+\left(t_{2}^{+}\right)^{2} \mu_{2}\left|u_{2}^{+}\right|_{4}^{4}+\left(t_{2}^{-}\right)^{2} \mu_{2}\left|u_{2}^{-}\right|_{4}^{4}\right] \\
& +\frac{1}{2}|\beta| \int_{\Omega}\left|\sqrt{t_{1}^{+}} u_{1}^{+}-\sqrt{t_{1}^{-}} u_{1}^{-}\right|^{2}\left|\sqrt{t_{2}^{+}} u_{2}^{+}-\sqrt{t_{2}^{-}} u_{2}^{-}\right|^{2} \\
\leq & \sum_{i=1}^{2}\left(\frac{t_{i}^{+}}{2}-\frac{\left(t_{i}^{+}\right)^{2}}{4}\right)\left\|u_{i}^{+}\right\|_{\lambda_{i}}^{2}+\sum_{i=1}^{2}\left(\frac{t_{i}^{-}}{2}-\frac{\left(t_{i}^{-}\right)^{2}}{4}\right)\left\|u_{i}^{-}\right\|_{\lambda_{i}}^{2} \\
\leq & \frac{1}{4}\left(\left\|u_{1}^{+}\right\|_{\lambda_{1}}^{2}+\left\|u_{1}^{-}\right\|_{\lambda_{1}}^{2}+\left\|u_{2}^{+}\right\|_{\lambda_{2}}^{2}+\left\|u_{2}^{-}\right\|_{\lambda_{2}}^{2}\right)=E_{\beta}\left(u_{1}, u_{2}\right) .
\end{aligned}
$$

Letting $\left(t_{1}^{+}, t_{1}^{-}, t_{2}^{+}, t_{2}^{-}\right)=(1,1,1,1)$, we completes the proof.

Lemma 3.2. $c=\tilde{c}$ and so $\vec{U}$ is a least energy sign-changing solution of (1.2).

Proof. Take any $\vec{u}=\left(u_{1}, u_{2}\right) \in \mathcal{N}_{\beta}$ such that $E_{\beta}(\vec{u})<c^{2,2}+1$. We define

$$
A:=A_{1} \times A_{2} ; \quad A_{i}:=\left\{u \in \operatorname{span}\left\{u_{i}^{+}, u_{i}^{-}\right\}:|u|_{4}=1\right\} .
$$

As in the proof of Lemma 2.9, one has $A \in \Gamma^{(2,2)}$. For any $\vec{v}=\left(v_{1}, v_{2}\right) \in A$, there exist $b_{i}, d_{i} \in \mathbb{R}$ such that $v_{i}=b_{i} u_{i}^{+}+d_{i} u_{i}^{-}$. Then by (2.10) and Lemma 3.1 we have

$$
\begin{aligned}
J_{\beta}(\vec{v}) & =\sup _{t_{1}, t_{2} \geq 0} E_{\beta}\left(\sqrt{t_{1}} v_{1}, \sqrt{t_{2}} v_{2}\right) \\
& =\sup _{t_{1}, t_{2} \geq 0} E_{\beta}\left(\sqrt{t_{1}}\left(b_{1} u_{1}^{+}+d_{1} u_{1}^{-}\right), \sqrt{t_{2}}\left(b_{2} u_{2}^{+}+d_{2} u_{2}^{-}\right)\right) \\
& =\sup _{t_{1}, t_{2} \geq 0} E_{\beta}\left(\sqrt{t_{1}}\left|b_{1}\right| u_{1}^{+}-\sqrt{t_{1}}\left|d_{1}\right| u_{1}^{-}, \sqrt{t_{2}}\left|b_{2}\right| u_{2}^{+}-\sqrt{t_{2}}\left|d_{2}\right| u_{2}^{-}\right) \\
& \leq E_{\beta}\left(u_{1}, u_{2}\right)
\end{aligned}
$$

that is, $\sup _{A} J_{\beta} \leq E_{\beta}(\vec{u})<c^{2,2}+1$ and so $A \in \Gamma_{\beta}^{(2,2)}$, which implies

$$
c=c_{\beta, \delta}^{2,2} \leq \sup _{\vec{v} \in A \backslash \mathcal{P}_{\delta}} J_{\beta}(\vec{v}) \leq E_{\beta}(\vec{u}), \quad \forall \vec{u} \in \mathcal{N}_{\beta} \text { with } E_{\beta}(\vec{u})<c^{2,2}+1
$$

Hence $c \leq \tilde{c}$, that is, $\tilde{c}=c=E_{\beta}(\vec{U})$. Since any sign-changing solutions belong to $\mathcal{N}_{\beta}$, so $\vec{U}=\left(U_{1}, U_{2}\right)$ is a least energy sign-changing solution of (1.2). 
To continue our proof, we need a classical result by Miranda.

Lemma 3.3 ([23]). Consider a rectangle $\mathbf{R}=\prod_{1}^{s}\left[a_{i}, b_{i}\right] \subset \mathbb{R}^{s}$ and a continuous function $\Phi: \mathbf{R} \rightarrow \mathbb{R}^{s}, \Phi=\left(\Phi_{1}, \ldots, \Phi_{s}\right)$. If $\left.\Phi_{i}\right|_{x_{i}=a_{i}}>0>\left.\Phi_{i}\right|_{x_{i}=b_{i}}$ holds for every $i$, then $\Phi$ has a zero inside $\mathbf{R}$.

Lemma 3.4. Both $U_{1}$ and $U_{2}$ has exactly two nodal domains.

Proof. Since $U_{1}, U_{2}$ both change sign, so both $U_{1}$ and $U_{2}$ have at least two nodal domains. Assume by contradiction that $U_{1}$ has at least three nodal domains $\Omega_{1}, \Omega_{2}$ and $\Omega_{3}$. Without loss of generality, we assume that $U_{1}>0$ on $\Omega_{1} \cup \Omega_{2}$. Define

$$
u_{1}^{+}:=\chi_{\Omega_{1}} U_{1}, \quad u_{1}^{-}:=\chi_{\Omega_{2}} U_{1}, \quad u_{3}:=\chi_{\Omega_{3}} U_{1},
$$

where

$$
\chi_{\Omega}(x):= \begin{cases}1, & x \in \Omega, \\ 0, & x \in \mathbb{R}^{N} \backslash \Omega .\end{cases}
$$

Then $u_{1}^{ \pm}, u_{3} \in H_{0}^{1}(\Omega) \backslash\{0\}$. By $E_{\beta}^{\prime}(\vec{U})\left(u_{1}^{ \pm}, 0\right)=0$ and $E_{\beta}^{\prime}(\vec{U})\left(0, U_{2}^{ \pm}\right)=0$ we have

$$
\begin{aligned}
& \left\|u_{1}^{ \pm}\right\|_{\lambda_{1}}^{2}=\mu_{1}\left|u_{1}^{ \pm}\right|_{4}^{4}-|\beta| \int_{\Omega}\left(u_{1}^{ \pm}\right)^{2} U_{2}^{2}, \\
& \left\|U_{2}^{ \pm}\right\|_{\lambda_{2}}^{2}=\mu_{2}\left|U_{2}^{ \pm}\right|_{4}^{4}-|\beta| \int_{\Omega} U_{1}^{2}\left(U_{2}^{ \pm}\right)^{2} .
\end{aligned}
$$

Let

$$
a:=\frac{1}{2} \min \left\{\frac{\left\|u_{1}^{ \pm}\right\|_{\lambda_{1}}^{2}}{\mu_{1}\left|u_{1}^{ \pm}\right|_{4}^{4}}, \quad \frac{\left\|U_{2}^{ \pm}\right\|_{\lambda_{2}}^{2}}{\mu_{2}\left|U_{2}^{ \pm}\right|_{4}^{4}}\right\}>0 .
$$

From (3.5)-(3.6) one has $a<1 / 2$. For any $b>1$, we define $\Phi=\left(f_{1}^{+}, f_{1}^{-}, f_{2}^{+}, f_{2}^{-}\right)$: $[a, b]^{4} \rightarrow \mathbb{R}^{4}$ by

$$
\begin{aligned}
& f_{1}^{ \pm}\left(t_{1}^{+}, t_{1}^{-}, t_{2}^{+}, t_{2}^{-}\right):=\left\|u_{1}^{ \pm}\right\|_{\lambda_{1}}^{2}-t_{1}^{ \pm} \mu_{1}\left|u_{1}^{ \pm}\right|_{4}^{4}+|\beta| \int_{\Omega}\left(u_{1}^{ \pm}\right)^{2}\left|\sqrt{t_{2}^{+}} U_{2}^{+}-\sqrt{t_{2}^{-}} U_{2}^{-}\right|^{2}, \\
& f_{2}^{ \pm}\left(t_{1}^{+}, t_{1}^{-}, t_{2}^{+}, t_{2}^{-}\right):=\left\|U_{2}^{ \pm}\right\|_{\lambda_{2}}^{2}-t_{2}^{ \pm} \mu_{2}\left|U_{2}^{ \pm}\right|_{4}^{4}+|\beta| \int_{\Omega}\left|\sqrt{t_{1}^{+}} u_{1}^{+}-\sqrt{t_{1}^{-}} u_{1}^{-}\right|^{2}\left(U_{2}^{ \pm}\right)^{2} .
\end{aligned}
$$

Then for any $\left(t_{1}^{+}, t_{1}^{-}, t_{2}^{+}, t_{2}^{-}\right) \in[a, b]^{4}$,

$$
\begin{aligned}
& \left.f_{1}^{ \pm}\right|_{t_{1}^{ \pm}=a} \geq\left\|u_{1}^{ \pm}\right\|_{\lambda_{1}}^{2}-a \mu_{1}\left|u_{1}^{ \pm}\right|_{4}^{4} \geq \frac{1}{2}\left\|u_{1}^{ \pm}\right\|_{\lambda_{1}}^{2}>0, \\
& \left.f_{2}^{ \pm}\right|_{t_{2}^{ \pm}=a} \geq\left\|U_{2}^{ \pm}\right\|_{\lambda_{2}}^{2}-a \mu_{2}\left|U_{2}^{ \pm}\right|_{4}^{4} \geq \frac{1}{2}\left\|U_{2}^{ \pm}\right\|_{\lambda_{2}}^{2}>0 .
\end{aligned}
$$


Moreover, by (3.5) we have

$$
\begin{aligned}
\left.f_{1}^{ \pm}\right|_{t_{1}^{ \pm}=b} & =\left\|u_{1}^{ \pm}\right\|_{\lambda_{1}}^{2}-b \mu_{1}\left|u_{1}^{ \pm}\right|_{4}^{4}+|\beta| \int_{\Omega}\left(u_{1}^{ \pm}\right)^{2}\left|\sqrt{t_{2}^{+}} U_{2}^{+}-\sqrt{t_{2}^{-}} U_{2}^{-}\right|^{2} \\
& =\left\|u_{1}^{ \pm}\right\|_{\lambda_{1}}^{2}-b \mu_{1}\left|u_{1}^{ \pm}\right|_{4}^{4}+|\beta| \int_{\Omega}\left(u_{1}^{ \pm}\right)^{2}\left(t_{2}^{+}\left(U_{2}^{+}\right)^{2}+t_{2}^{-}\left(U_{2}^{-}\right)^{2}\right) \\
& \leq\left\|u_{1}^{ \pm}\right\|_{\lambda_{1}}^{2}-b \mu_{1}\left|u_{1}^{ \pm}\right|_{4}^{4}+b|\beta| \int_{\Omega}\left(u_{1}^{ \pm}\right)^{2} U_{2}^{2} \\
& =(1-b)\left\|u_{1}^{ \pm}\right\|_{\lambda_{1}}^{2}<0 .
\end{aligned}
$$

Similarly, by (3.6) we have

$$
\begin{aligned}
\left.f_{2}^{ \pm}\right|_{t_{2}^{ \pm}=b} & \leq\left\|U_{2}^{ \pm}\right\|_{\lambda_{2}}^{2}-b \mu_{2}\left|U_{2}^{ \pm}\right|_{4}^{4}+b|\beta| \int_{\Omega} U_{1}^{2}\left(U_{2}^{ \pm}\right)^{2} \\
& =(1-b)\left\|U_{2}^{ \pm}\right\|_{\lambda_{2}}^{2}<0 .
\end{aligned}
$$

Then by Lemma 3.3 there exists $\left(\tilde{t}_{1}^{+}, \tilde{t}_{1}^{-}, \tilde{t}_{2}^{+}, \tilde{t}_{2}^{-}\right) \in[a, b]^{4}$ such that

$$
f_{1}^{ \pm}\left(\tilde{t}_{1}^{+}, \tilde{t}_{1}^{-}, \tilde{t}_{2}^{+}, \tilde{t}_{2}^{-}\right)=0, \quad f_{2}^{ \pm}\left(\tilde{t}_{1}^{+}, \tilde{t}_{1}^{-}, \tilde{t}_{2}^{+}, \tilde{t}_{2}^{-}\right)=0 .
$$

This implies that

$$
\left(\sqrt{\tilde{t}_{1}^{+}} u_{1}^{+}-\sqrt{\tilde{t}_{1}^{-}} u_{1}^{-}, \sqrt{\tilde{t}_{2}^{+}} U_{2}^{+}-\sqrt{\tilde{t}_{2}^{-}} U_{2}^{-}\right) \in \mathcal{N}_{\beta} .
$$

Remark that (3.7) and (3.8) hold for any $b>1$, so we obtain that

$$
\tilde{t}_{1}^{+} \leq 1, \quad \tilde{t}_{1}^{-} \leq 1, \quad \tilde{t}_{2}^{+} \leq 1, \quad \tilde{t}_{2}^{-} \leq 1
$$

Hence

$$
\begin{aligned}
c & \leq E_{\beta}\left(\sqrt{\tilde{t}_{1}^{+}} u_{1}^{+}-\sqrt{\tilde{t}_{1}^{-}} u_{1}^{-}, \sqrt{\tilde{t}_{2}^{+}} U_{2}^{+}-\sqrt{\tilde{t}_{2}^{-}} U_{2}^{-}\right) \\
& =\frac{1}{4}\left(\tilde{t}_{1}^{+}\left\|u_{1}^{+}\right\|_{\lambda_{1}}^{2}+\tilde{t}_{1}^{-}\left\|u_{1}^{-}\right\|_{\lambda_{1}}^{2}+\tilde{t}_{2}^{+}\left\|U_{2}^{+}\right\|_{\lambda_{2}}^{2}+\tilde{t}_{2}^{-}\left\|U_{2}^{-}\right\|_{\lambda_{2}}^{2}\right) \\
& <\frac{1}{4}\left(\left\|u_{1}^{+}\right\|_{\lambda_{1}}^{2}+\left\|u_{1}^{-}\right\|_{\lambda_{1}}^{2}+\left\|u_{3}\right\|_{\lambda_{1}}^{2}+\left\|U_{2}^{+}\right\|_{\lambda_{2}}^{2}+\left\|U_{2}^{-}\right\|_{\lambda_{2}}^{2}\right) \\
& \leq \frac{1}{4}\left(\left\|U_{1}\right\|_{\lambda_{1}}^{2}+\left\|U_{2}\right\|_{\lambda_{2}}^{2}\right)=E_{\beta}\left(U_{1}, U_{2}\right)=c,
\end{aligned}
$$

a contradiction. Hence $U_{1}$ has exactly two nodal domains. Similarly, $U_{2}$ has exactly two nodal domains.

Proof of Theorem 1.6. Theorem 1.6 follows directly from Lemmas 3.2 and 3.4. 


\section{Proof of Theorem 1.7}

The following arguments are similar to those in Sections 2-3 with some important modifications. Here, although some definitions are slight different from those in Section 2, we will use the same notation as in Section 2 for convenience. To obtain semi-nodal solutions $\left(u_{1}, u_{2}\right)$ such that $u_{1}$ changes sign and $u_{2}$ is positive, we consider the following functional

$$
\widetilde{E}_{\beta}\left(u_{1}, u_{2}\right):=\frac{1}{2}\left(\left\|u_{1}\right\|_{\lambda_{1}}^{2}+\left\|u_{2}\right\|_{\lambda_{2}}^{2}\right)-\frac{1}{4}\left(\mu_{1}\left|u_{1}\right|_{4}^{4}+\mu_{2}\left|u_{2}^{+}\right|_{4}^{4}\right)+\frac{|\beta|}{2} \int_{\Omega} u_{1}^{2} u_{2}^{2},
$$

and modify the definition of $\widetilde{H}$ by

$$
\widetilde{H}:=\left\{\left(u_{1}, u_{2}\right) \in H: u_{1} \neq 0, u_{2}^{+} \neq 0\right\} .
$$

Then by similar proofs as in Section 2, we have the following lemmas.

Lemma 4.1. For any $\left(u_{1}, u_{2}\right) \in \widetilde{H}$, if $|\beta|^{2}\left(\int_{\Omega} u_{1}^{2} u_{2}^{2}\right)^{2} \geq \mu_{1} \mu_{2}\left|u_{1}\right|_{4}^{4}\left|u_{2}^{+}\right|_{4}^{4}$, then

$$
\sup _{t_{1}, t_{2} \geq 0} \widetilde{E}_{\beta}\left(\sqrt{t_{1}} u_{1}, \sqrt{t_{2}} u_{2}\right)=+\infty \text {. }
$$

Lemma 4.2. For any $\vec{u}=\left(u_{1}, u_{2}\right) \in \widetilde{H}$, if

$$
|\beta|^{2}\left(\int_{\Omega} u_{1}^{2} u_{2}^{2}\right)^{2}<\mu_{1} \mu_{2}\left|u_{1}\right|_{4}^{4}\left|u_{2}^{+}\right|_{4}^{4}
$$

then system

$$
\left\{\begin{array}{l}
\left\|u_{1}\right\|_{\lambda_{1}}^{2}=t_{1} \mu_{1}\left|u_{1}\right|_{4}^{4}-t_{2}|\beta| \int_{\Omega} u_{1}^{2} u_{2}^{2} \\
\left\|u_{2}\right\|_{\lambda_{2}}^{2}=t_{2} \mu_{2}\left|u_{2}^{+}\right|_{4}^{4}-t_{1}|\beta| \int_{\Omega} u_{1}^{2} u_{2}^{2}
\end{array}\right.
$$

has a unique solution

$$
\left\{\begin{array}{l}
t_{1}(\vec{u})=\frac{\mu_{2}\left|u_{2}^{+}\right|_{4}^{4}\left\|u_{1}\right\|_{\lambda_{1}}^{2}+|\beta|\left\|u_{2}\right\|_{\lambda_{2}}^{2} \int_{\Omega} u_{1}^{2} u_{2}^{2}}{\mu_{1} \mu_{2}\left|u_{1}\right|_{4}^{4}\left|u_{2}^{+}\right|_{4}^{4}-|\beta|^{2}\left(\int_{\Omega} u_{1}^{2} u_{2}^{2}\right)^{2}}>0 \\
t_{2}(\vec{u})=\frac{\mu_{1}\left|u_{1}\right|_{4}^{4}\left\|u_{2}\right\|_{\lambda_{2}}^{2}+|\beta||| u_{1} \|_{\lambda_{1}}^{2} \int_{\Omega} u_{1}^{2} u_{2}^{2}}{\mu_{1} \mu_{2}\left|u_{1}\right|_{4}^{4}\left|u_{2}^{+}\right|_{4}^{4}-|\beta|^{2}\left(\int_{\Omega} u_{1}^{2} u_{2}^{2}\right)^{2}}>0 .
\end{array}\right.
$$

Moreover,

$$
\begin{aligned}
\sup _{t_{1}, t_{2} \geq 0} & \widetilde{E}_{\beta}\left(\sqrt{t_{1}} u_{1}, \sqrt{t_{2}} u_{2}\right)=\widetilde{E}_{\beta}\left(\sqrt{t_{1}(\vec{u})} u_{1}, \sqrt{t_{2}(\vec{u})} u_{2}\right) \\
= & \frac{1}{4} \frac{\mu_{2}\left|u_{2}^{+}\right|_{4}^{4}\left\|u_{1}\right\|_{\lambda_{1}}^{4}+2|\beta|\left\|u_{1}\right\|_{\lambda_{1}}^{2}\left\|u_{2}\right\|_{\lambda_{2}}^{2} \int_{\Omega} u_{1}^{2} u_{2}^{2}+\mu_{1}\left|u_{1}\right|_{4}^{4}\left\|u_{2}\right\|_{\lambda_{2}}^{4}}{\mu_{1} \mu_{2}\left|u_{1}\right|_{4}^{4}\left|u_{2}^{+}\right|_{4}^{4}-|\beta|^{2}\left(\int_{\Omega} u_{1}^{2} u_{2}^{2}\right)^{2}}
\end{aligned}
$$

and $\left(t_{1}(\vec{u}), t_{2}(\vec{u})\right)$ is the unique maximum point of $\widetilde{E}_{\beta}\left(\sqrt{t_{1}} u_{1}, \sqrt{t_{2}} u_{2}\right)$. 
Now, we modify the definitions of $\mathcal{M}^{*}, \mathcal{M}_{\beta}^{*}, \mathcal{M}_{\beta}^{* *}, \mathcal{M}$ and $\mathcal{M}_{\beta}$ by

$$
\begin{aligned}
\mathcal{M}^{*} & :=\left\{\vec{u} \in H:\left|u_{1}\right|_{4}>1 / 2,\left|u_{2}^{+}\right|_{4}>1 / 2\right\} \\
\mathcal{M}_{\beta}^{*} & :=\left\{\vec{u} \in \mathcal{M}^{*}: \vec{u} \text { satisfies }(4.1)\right\} \\
\mathcal{M}_{\beta}^{* *} & :=\left\{\vec{u} \in \mathcal{M}^{*}: \mu_{1} \mu_{2}-|\beta|^{2}\left(\int_{\Omega} u_{1}^{2} u_{2}^{2}\right)^{2}>0\right\} ; \\
\mathcal{M} & :=\left\{\vec{u} \in H:\left|u_{1}\right|_{4}=1,\left|u_{2}^{+}\right|_{4}=1\right\}, \quad \mathcal{M}_{\beta}:=\mathcal{M} \cap \mathcal{M}_{\beta}^{*},
\end{aligned}
$$

and define a new functional $J_{\beta}: \mathcal{M}^{*} \rightarrow(0,+\infty]$ as in Section 2 by

$$
J_{\beta}(\vec{u}):= \begin{cases}\frac{1}{4} \frac{\mu_{2}\left\|u_{1}\right\|_{\lambda_{1}}^{4}+2|\beta|\left\|u_{1}\right\|_{\lambda_{1}}^{2}\left\|u_{2}\right\|_{\lambda_{2}}^{2} \int_{\Omega} u_{1}^{2} u_{2}^{2}+\mu_{1}\left\|u_{2}\right\|_{\lambda_{2}}^{4}}{\mu_{1} \mu_{2}-|\beta|^{2}\left(\int_{\Omega} u_{1}^{2} u_{2}^{2}\right)^{2}} & \text { if } \vec{u} \in \mathcal{M}_{\beta}^{* *}, \\ +\infty & \text { if } \vec{u} \in \mathcal{M}^{*} \backslash \mathcal{M}_{\beta}^{* *}\end{cases}
$$

Then $J_{\beta} \in C\left(\mathcal{M}^{*},(0,+\infty]\right), \inf _{\mathcal{M}^{*}} J_{\beta} \geq C_{1}>0$ where $C_{1}$ independent of $\beta<$ $0, J_{\beta} \in C^{1}\left(\mathcal{M}_{\beta}^{* *},(0,+\infty)\right)$ and (2.8)-(2.9) hold for any $\vec{u} \in \mathcal{M}_{\beta}$ and $\varphi, \psi \in$ $H_{0}^{1}(\Omega)$. Note that Lemmas 4.1 and 4.2 yield

$$
J_{\beta}\left(u_{1}, u_{2}\right)=\sup _{t_{1}, t_{2} \geq 0} \widetilde{E}_{\beta}\left(\sqrt{t_{1}} u_{1}, \sqrt{t_{2}} u_{2}\right), \quad \forall\left(u_{1}, u_{2}\right) \in \mathcal{M}
$$

For any $\vec{u}=\left(u_{1}, u_{2}\right) \in \mathcal{M}_{\beta}^{*}$, let $\tilde{w}_{i} \in H_{0}^{1}(\Omega), i=1,2$, be the unique solutions of the following linear problem

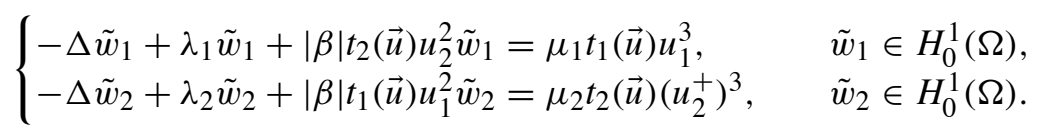

As in Section 2, we define

$$
w_{i}=\alpha_{i} \tilde{w}_{i}, \quad \text { where } \alpha_{1}=\frac{1}{\int_{\Omega} u_{1}^{3} \tilde{w}_{1}}>0, \alpha_{2}=\frac{1}{\int_{\Omega}\left(u_{2}^{+}\right)^{3} \tilde{w}_{2}}>0
$$

Then $\left(w_{1}, w_{2}\right)$ is the unique solution of the problem

$$
\begin{cases}-\Delta w_{1}+\lambda_{1} w_{1}+|\beta| t_{2}(\vec{u}) u_{2}^{2} w_{1}=\alpha_{1} \mu_{1} t_{1}(\vec{u}) u_{1}^{3}, & w_{1} \in H_{0}^{1}(\Omega), \\ -\Delta w_{2}+\lambda_{2} w_{2}+|\beta| t_{1}(\vec{u}) u_{1}^{2} w_{2}=\alpha_{2} \mu_{2} t_{2}(\vec{u})\left(u_{2}^{+}\right)^{3}, & w_{2} \in H_{0}^{1}(\Omega), \\ \int_{\Omega} u_{1}^{3} w_{1} d x=1, \quad \int_{\Omega}\left(u_{2}^{+}\right)^{3} w_{2} d x=1 . & \end{cases}
$$

As in Section 2, the operator $K=\left(K_{1}, K_{2}\right): \mathcal{M}_{\beta}^{*} \rightarrow H$ is defined as $K(\vec{u}):=$ $\vec{w}=\left(w_{1}, w_{2}\right)$, and similar arguments as Lemma 2.4 yield $K \in C^{1}\left(\mathcal{M}_{\beta}^{*}, H\right)$. Since 
$u_{n} \rightarrow u$ in $L^{4}(\Omega)$ implies $u_{n}^{+} \rightarrow u^{+}$in $L^{4}(\Omega)$, so Lemma 2.5 and its proof with obvious modifications also hold for this new $K$ defined here. Note that

$$
K\left(\sigma_{1}(\vec{u})\right)=\sigma_{1}(K(\vec{u})) .
$$

Remark that (4.10) only holds for $\sigma_{1}$ and in the sequel we only use $\sigma_{1}$. Consider

$$
\mathcal{F}=\left\{A \subset \mathcal{M}: A \text { is closed and } \sigma_{1}(\vec{u}) \in A \forall \vec{u} \in A\right\},
$$

and, for each $A \in \mathcal{F}$ and $k_{1} \geq 2$, the class of functions

$$
F_{\left(k_{1}, 1\right)}(A)=\left\{f: A \rightarrow \mathbb{R}^{k_{1}-1}: f \text { continuous and } f\left(\sigma_{1}(\vec{u})\right)=-f(\vec{u})\right\} .
$$

Definition 4.3 (Modified vector genus, slightly different from Definition 2.6). Let $A \in \mathcal{F}$ and take any $k_{1} \in \mathbb{N}$ with $k_{1} \geq 2$. We say that $\vec{\gamma}(A) \geq\left(k_{1}, 1\right)$ if for every $f \in F_{\left(k_{1}, 1\right)}(A)$ there exists $\vec{u} \in A$ such that $f(\vec{u})=0$. We denote

$$
\Gamma^{\left(k_{1}, 1\right)}:=\left\{A \in \mathcal{F}: \vec{\gamma}(A) \geq\left(k_{1}, 1\right)\right\}
$$

Lemma 4.4. With the previous notations, the following properties hold.

(i) Take $A:=A_{1} \times A_{2} \subset \mathcal{M}$ and let $\eta: S^{k_{1}-1} \rightarrow A_{1}$ be a homeomorphism such that $\eta(-x)=-\eta(x)$ for every $x \in S^{k_{1}-1}$. Then $A \in \Gamma^{\left(k_{1}, 1\right)}$.

(ii) We have $\overline{\eta(A)} \in \Gamma^{\left(k_{1}, 1\right)}$ whenever $A \in \Gamma^{\left(k_{1}, 1\right)}$ and a continuous map $\eta: A \rightarrow$ $\mathcal{M}$ is such that $\eta \circ \sigma_{1}=\sigma_{1} \circ \eta$.

Proof. The conclusion (ii) is trivial, we only prove (i). Fix any $f \in F_{\left(k_{1}, 1\right)}(A)$ and take any $u_{2} \in A_{2}$. Define $\varphi: S^{k_{1}-1} \rightarrow \mathbb{R}^{k_{1}-1}$ by $\varphi(x):=f\left(\eta(x), u_{2}\right)$. Then $\varphi$ is continuous and $\varphi(-x)=-\varphi(x)$. So by Borsuk-Ulam Theorem, there exists $x_{0} \in S^{k_{1}-1}$ such that $\varphi\left(x_{0}\right)=0$, that is $f\left(\eta\left(x_{0}\right), u_{2}\right)=0$. Hence $\vec{\gamma}(A) \geq\left(k_{1}, 1\right)$ and $A \in \Gamma^{\left(k_{1}, 1\right)}$.

Now we modify the definitions of $\mathcal{P}$ and $\operatorname{dist}_{4}(\vec{u}, \mathcal{P})$ in (2.19)-(2.20) by

$$
\mathcal{P}:=\mathcal{P}_{1} \cup-\mathcal{P}_{1}, \quad \operatorname{dist}_{4}(\vec{u}, \mathcal{P}):=\min \left\{\operatorname{dist}_{4}\left(u_{1}, \mathcal{P}_{1}\right), \operatorname{dist}_{4}\left(u_{1},-\mathcal{P}_{1}\right)\right\} .
$$

Under this new definition, $u_{1}$ changes sign if $\operatorname{dist}_{4}(\vec{u}, \mathcal{P})>0$.

Lemma 4.5. Let $k_{1} \geq 2$. Then for any $\delta<2^{-1 / 4}$ and any $A \in \Gamma^{\left(k_{1}, 1\right)}$ there holds $A \backslash \mathcal{P}_{\delta} \neq \emptyset$.

Proof. Fix any $A \in \Gamma^{\left(k_{1}, 1\right)}$. Recall the map $f_{1}: A \rightarrow \mathbb{R}^{k_{1}-1}$ defined in (2.21). Clearly $f_{1} \in F_{\left(k_{1}, 1\right)}(A)$, so there exists $\vec{u} \in A$ such that $f_{1}(\vec{u})=0$, which means $\int_{\Omega}\left(u_{1}^{+}\right)^{4}=\int_{\Omega}\left(u_{1}^{-}\right)^{4}=1 / 2$, that is, $\operatorname{dist}_{4}(\vec{u}, \mathcal{P})=2^{-1 / 4}$, so $\vec{u} \in A \backslash \mathcal{P}_{\delta}$ for every $\delta<2^{-1 / 4}$.

Lemma 4.6. Let $k_{1} \geq 2$. There exist $A \in \Gamma^{\left(k_{1}, 1\right)}$ and a constant $c^{k_{1}, 1} \in \mathbb{N}$ independent of $\beta<0$ such that $\sup _{A} J_{\beta} \leq c^{k_{1}, 1}$ for any $\beta<0$. 
Proof. Let $B_{i}$ and $\left\{\varphi_{k}^{i}: 1 \leq k \leq k_{i}\right\} \subset H_{0}^{1}\left(B_{i}\right)$ be in the proof of Lemma 2.9. Define

$$
A_{1}:=\left\{u \in \operatorname{span}\left\{\varphi_{1}^{1}, \ldots, \varphi_{k_{1}}^{1}\right\}:|u|_{4}=1\right\}, \quad A_{2}=\left\{C\left|\varphi_{1}^{2}\right|: C=1 /\left|\varphi_{1}^{2}\right|_{4}\right\} .
$$

Then by Lemma 4.4-(i) one has $A:=A_{1} \times A_{2} \in \Gamma^{\left(k_{1}, 1\right)}$. The rest of the proof is the same as Lemma 2.9 .

For every $k_{1} \geq 2$ and $0<\delta<2^{-1 / 4}$, we define

$$
c_{\beta, \delta}^{k_{1}, 1}:=\inf _{A \in \Gamma_{\beta}^{\left(k_{1}, 1\right)}} \sup _{\vec{u} \in A \backslash \mathcal{P}_{\delta}} J_{\beta}(\vec{u}),
$$

where the definition of $\Gamma_{\beta}^{\left(k_{1}, 1\right)}$ is the same as (2.23). Then Lemma 4.6 yields $\Gamma_{\beta}^{\left(k_{1}, 1\right)} \neq \emptyset$ and so $c_{\beta, \delta}^{k_{1}, 1}$ is well defined. Moreover, $c_{\beta, \delta}^{k_{1}, 1} \leq c^{k_{1}, 1}$ for any $\beta<0$ and $\delta>0$. Under the new definitions (4.11), it is easy to see that Lemma 2.10 also holds here. Now as in Section 2, we define a map $V: \mathcal{M}_{\beta}^{*} \rightarrow H$ by $V(\vec{u}):=\vec{u}-K(\vec{u})$. Then Lemma 2.11 also holds here. Recall from (4.5) and (4.9) that

$$
\int_{\Omega}\left(u_{2}^{+}\right)^{3}\left(u_{2}-w_{2}\right) d x=1-1=0, \quad \forall \vec{u}=\left(u_{1}, u_{2}\right) \in \mathcal{M}_{\beta} .
$$

Then by similar arguments, we see that Lemma 2.12 also holds here.

Lemma 4.7. There exists a unique global solution $\eta=\left(\eta_{1}, \eta_{2}\right):[0, \infty) \times \mathcal{M}_{\beta} \rightarrow$ $H$ for the initial value problem

$$
\frac{d}{d t} \eta(t, \vec{u})=-V(\eta(t, \vec{u})), \quad \eta(0, \vec{u})=\vec{u} \in \mathcal{M}_{\beta} .
$$

Moreover, conclusions (i), (iii) and (iv) of Lemma 2.13 also hold here, and $\eta\left(t, \sigma_{1}(\vec{u})\right)=\sigma_{1}(\eta(t, \vec{u}))$ for any $t>0$ and $u \in \mathcal{M}_{\beta}$.

Proof. Recalling $V(\vec{u}) \in C^{1}\left(\mathcal{M}_{\beta}^{*}, H\right),(4.12)$ has a unique solution $\eta:\left[0, T_{\max }\right) \times$ $\mathcal{M}_{\beta} \rightarrow H$, where $T_{\max }>0$ is the maximal time such that $\eta(t, \vec{u}) \in \mathcal{M}_{\beta}^{*}$ for all $t \in\left[0, T_{\max }\right)$. Fixed any $\vec{u}=\left(u_{1}, u_{2}\right) \in \mathcal{M}_{\beta}$, we deduce from (4.12) that

$$
\begin{aligned}
\frac{d}{d t} \int_{\Omega}\left(\eta_{2}(t, \vec{u})^{+}\right)^{4} d x & =-4 \int_{\Omega}\left(\eta_{2}(t, \vec{u})^{+}\right)^{3}\left(\eta_{2}(t, \vec{u})-K_{2}(\eta(t, \vec{u}))\right) d x \\
& =4-4 \int_{\Omega}\left(\eta_{2}(t, \vec{u})^{+}\right)^{4} d x, \quad \forall 0<t<T_{\max },
\end{aligned}
$$

that is

$$
\frac{d}{d t}\left[e^{4 t}\left(\int_{\Omega}\left(\eta_{2}(t, \vec{u})^{+}\right)^{4} d x-1\right)\right]=0 .
$$

Since $\int_{\Omega}\left(\eta_{2}(0, \vec{u})^{+}\right)^{4} d x=\int_{\Omega}\left(u_{2}^{+}\right)^{4} d x=1$, so $\int_{\Omega}\left(\eta_{2}(t, \vec{u})^{+}\right)^{4} d x \equiv 1$ for all $0 \leq t<T_{\max }$. Recalling (4.10), the rest of the proof is similar to Lemma 2.13. 
Now we can give the proof of Theorem 1.7.

Proof of Theorem 1.7.

Step 1. Fix any $k_{1} \geq 2$. We prove that $c_{\beta, \delta}^{k_{1}, 1}$ is a sign-changing critical value of $E_{\beta}$ for $\delta>0$ small.

By similar arguments as Step 1 in the proof of Theorem 1.3, for small $\delta>0$, there exists $\vec{u}=\left(u_{1}, u_{2}\right) \in \mathcal{M}_{\beta}$ such that

$$
J_{\beta}(\vec{u})=c_{\beta, \delta}^{k_{1}, 1}, \quad V(\vec{u})=0 \quad \text { and } \operatorname{dist}_{4}(\vec{u}, \mathcal{P}) \geq \delta .
$$

Then $u_{1}$ changes sign. Since $V(\vec{u})=0$, so $\vec{u}=K(\vec{u})$. Combining this with (4.9), we see that $\vec{u}$ satisfies

$$
\left\{\begin{array}{l}
-\Delta u_{1}+\lambda_{1} u_{1}+|\beta| t_{2}(\vec{u}) u_{2}^{2} u_{1}=\alpha_{1} \mu_{1} t_{1}(\vec{u}) u_{1}^{3}, \\
-\Delta u_{2}+\lambda_{2} u_{2}+|\beta| t_{1}(\vec{u}) u_{1}^{2} u_{2}=\alpha_{2} \mu_{2} t_{2}(\vec{u})\left(u_{2}^{+}\right)^{3} .
\end{array}\right.
$$

Since $\left|u_{1}\right|_{4}=1,\left|u_{2}^{+}\right|_{4}=1$ and $t_{i}(\vec{u})$ satisfies (4.2), so $\alpha_{1}=\alpha_{2}=1$. Multiply the second equation of (4.13) by $u_{2}^{-}$and integrate over $\Omega$, we get $\left\|u_{2}^{-}\right\|_{\lambda_{2}}^{2}=0$, so $u_{2} \geq 0$. By the strong maximum principle, $u_{2}>0$ in $\Omega$. Hence $\left(\tilde{u}_{1}, \tilde{u}_{2}\right):=$ $\left(\sqrt{t_{1}(\vec{u})} u_{1}, \sqrt{t_{2}(\vec{u})} u_{2}\right)$ is a semi-nodal solution of the original problem (1.2) with $\tilde{u}_{1}$ sign-changing and $\tilde{u}_{2}$ positive. Moreover, (4.4) and (4.6) yield

$$
E_{\beta}\left(\tilde{u}_{1}, \tilde{u}_{2}\right)=\widetilde{E}_{\beta}\left(\tilde{u}_{1}, \tilde{u}_{2}\right)=J_{\beta}\left(u_{1}, u_{2}\right)=c_{\beta, \delta}^{k_{1}, 1} \leq c^{k_{1}, 1} .
$$

Step 2. We prove that (1.2) has infinitely many semi-nodal solutions.

Assume by contradiction that there exist $k_{1}^{n} \rightarrow \infty, \delta_{n} \in\left(0,2^{-5 / 4}\right]$ and a positive constant $C$ such that $c_{\beta, \delta_{n}}^{k_{1}^{n}, 1} \leq C$ for every $n \in \mathbb{N}$. Then there exists $A_{n} \in$ $\Gamma_{\beta}^{\left(k_{1}^{n}, 1\right)}$ such that $\sup _{A_{n} \backslash \mathcal{P}_{\delta_{n}}} J_{\beta} \leq C+1$ for any $n \in \mathbb{N}$. Let $\left\{\varphi_{k}\right\}_{k} \subset H_{0}^{1}(\Omega)$ be as in the proof of Theorem 1.3 and recall the map $g_{1}^{n}: A_{n} \rightarrow \mathbb{R}^{k_{1}^{n}-1}$ defined in (2.31). Then $g_{1}^{n} \in F_{\left(k_{1}^{n}, 1\right)}\left(A_{n}\right)$. By the same arguments as in the proof of Theorem 1.3, we get a contradiction. Therefore, (1.2) has infinitely many semi-nodal solutions $\left\{\vec{u}_{n}=\left(u_{n, 1}, u_{n, 2}\right)\right\}_{n \geq 2}$ which satisfy

(1) $u_{n, 1}$ changes sign and $u_{n, 2}$ is positive;

(2) $E_{\beta}\left(u_{n, 1}, u_{n, 2}\right)=c_{\beta, \delta_{n}}^{n, 1} \leq c^{n, 1}$ for some $0<\delta_{n}<2^{-1 / 4}$. Moreover,

$$
\left\|u_{n, 1}\right\|_{L^{\infty}(\Omega)}+\left\|u_{n, 2}\right\|_{L^{\infty}(\Omega)} \rightarrow+\infty \quad \text { as } n \rightarrow+\infty .
$$

Step 3. We prove that $u_{n, 1}$ has at most $n$ nodal domains.

Assume that $u_{n, 1}$ has at least $n+1$ nodal domains $\Omega_{k}, 1 \leq k \leq n+1$, then $u_{n, 1} \chi \Omega_{k} \in H_{0}^{1}(\Omega)$. For $1 \leq k \leq n$, we see from $E_{\beta}^{\prime}\left(\vec{u}_{n}\right)\left(u_{n, 1} \chi_{\Omega_{k}}, 0\right)=0$ that

$$
\mu_{1}\left|u_{n, 1} \chi \Omega_{k}\right|_{4}^{4}=\left\|u_{n, 1} \chi_{\Omega_{k}}\right\|_{\lambda_{1}}^{2}+|\beta| \int_{\Omega}\left|u_{n, 1} \chi_{\Omega_{k}}\right|^{2} u_{n, 2}^{2}, \quad k=1, \ldots, n .
$$


Similarly $E_{\beta}^{\prime}\left(\vec{u}_{n}\right)\left(0, u_{n, 2}\right)=0$ yields

$$
\mu_{2}\left|u_{n, 2}\right|_{4}^{4}=\left\|u_{n, 2}\right\|_{\lambda_{2}}^{2}+|\beta| \int_{\Omega} u_{n, 1}^{2} u_{n, 2}^{2} .
$$

Then, similarly as Lemma 3.1 we have

$$
\begin{aligned}
& 2|\beta| \int_{\Omega}\left|\sum_{k=1}^{n} \sqrt{t_{k}} u_{n, 1} \chi_{\Omega_{k}}\right|^{2}\left|\sqrt{s} u_{n, 2}\right|^{2} \\
\leq & \sum_{k=1}^{n}|\beta| t_{k}^{2} \int_{\Omega}\left|u_{n, 1} \chi_{\Omega_{k}}\right|^{2} u_{n, 2}^{2}+|\beta| s^{2} \int_{\Omega} u_{n, 1}^{2} u_{n, 2}^{2} \\
\leq & \sum_{k=1}^{n} t_{k}^{2}\left(\mu_{1}\left|u_{n, 1} \chi_{\Omega_{k}}\right|_{4}^{4}-\left\|u_{n, 1} \chi_{\Omega_{k}}\right\|_{\lambda_{1}}^{2}\right)+s^{2}\left(\mu_{n, 2}\left|u_{n, 2}\right|_{4}^{4}-\left\|u_{n, 2}\right\|_{\lambda_{2}}^{2}\right) .
\end{aligned}
$$

Recall that $u_{n, 2}$ is positive, so for $t_{1}, \ldots, t_{n}, s \geq 0$,

$$
\begin{aligned}
& \widetilde{E}_{\beta}\left(\sum_{k=1}^{n} \sqrt{t_{k}} u_{n, 1} \chi_{\Omega_{k}}, \sqrt{s} u_{n, 2}\right) \\
=\sum_{k=1}^{n}\left(\frac{t_{k}}{2}\left\|u_{n, 1} \chi_{\Omega_{k}}\right\|_{\lambda_{1}}^{2}-\frac{t_{k}^{2}}{4} \mu_{1}\left|u_{n, 1} \chi_{\Omega_{k}}\right|_{4}^{4}\right) & +\left(\frac{s}{2}\left\|u_{n, 2}\right\|_{\lambda_{2}}^{2}-\frac{s^{2}}{4} \mu_{2}\left|u_{n, 2}\right|_{4}^{4}\right) \\
& +\frac{|\beta|}{2} \int_{\Omega}\left|\sum_{k=1}^{n} \sqrt{t_{k}} u_{n, 1} \chi_{\Omega_{k}}\right|^{2}\left|\sqrt{s} u_{n, 2}\right|^{2} \\
= & \sum_{k=1}^{n}\left(\frac{t_{k}}{2}-\frac{t_{k}^{2}}{4}\right)\left\|u_{n, 1} \chi_{\Omega_{k}}\right\|_{\lambda_{1}}^{2}+\left(\frac{s}{2}-\frac{s^{2}}{4}\right)\left\|u_{n, 2}\right\|_{\lambda_{2}}^{2} \\
\leq & \frac{1}{4} \sum_{k=1}^{n}\left\|u_{n, 1} \chi_{\Omega_{k}}\right\|_{\lambda_{1}}^{2}+\frac{1}{4}\left\|u_{n, 2}\right\|_{\lambda_{2}}^{2} .
\end{aligned}
$$

Now we define

$$
\begin{aligned}
A & :=A_{1} \times\left\{C u_{n, 2}: C=1 /\left|u_{n, 2}\right|_{4}\right\} ; \\
A_{1} & :=\left\{u \in \operatorname{span}\left\{u_{n, 1} \chi_{\Omega_{1}}, \ldots, u_{n, 1} \chi_{\Omega_{n}}\right\}:|u|_{4}=1\right\} .
\end{aligned}
$$

Then Lemma 4.4-(i) yields $A \in \Gamma^{(n, 1)}$, and similarly as Lemma 3.2, we deduce from (4.6) and (4.15) that

$$
\begin{aligned}
\sup _{A} J_{\beta} & \leq \frac{1}{4} \sum_{k=1}^{n}\left\|u_{n, 1} \chi_{\Omega_{k}}\right\|_{\lambda_{1}}^{2}+\frac{1}{4}\left\|u_{n, 2}\right\|_{\lambda_{2}}^{2}<\frac{1}{4}\left(\left\|u_{n, 1}\right\|_{\lambda_{1}}^{2}+\left\|u_{n, 2}\right\|_{\lambda_{2}}^{2}\right) \\
& =E_{\beta}\left(\vec{u}_{n}\right)=c_{\beta, \delta_{n}}^{n, 1} \leq c^{n, 1},
\end{aligned}
$$


and so $A \in \Gamma_{\beta}^{(n, 1)}$, which implies

$$
c_{\beta, \delta_{n}}^{n, 1} \leq \sup _{A \backslash \mathcal{P}_{\delta_{n}}} J_{\beta} \leq \frac{1}{4} \sum_{k=1}^{n}\left\|u_{n, 1} \chi_{\Omega_{k}}\right\|_{\lambda_{1}}^{2}+\frac{1}{4}\left\|u_{n, 2}\right\|_{\lambda_{2}}^{2}<E_{\beta}\left(\vec{u}_{n}\right),
$$

a contradiction. Hence $u_{n, 1}$ has at most $n$ nodal domains. In particular, $u_{2,1}$ has exactly two nodal domains.

Step 4. We prove that $\left(u_{2,1}, u_{2,2}\right)$ has the least energy among all nontrivial solutions whose first component changes sign.

By similar arguments as in Section 3, we can prove that

$$
c_{\beta, \delta_{2}}^{2,1}=\inf _{\vec{u} \in \mathcal{N}_{2,1, \beta}} E_{\beta}(\vec{u})=\inf _{\vec{u} \in \mathcal{N}_{2,1, \beta}} \widetilde{E}_{\beta}(\vec{u})
$$

where

$$
\begin{gathered}
\mathcal{N}_{2,1, \beta}:=\left\{\vec{u}=\left(u_{1}, u_{2}\right) \in H: u_{1} \text { changes sign and } u_{2} \geq 0, u_{2} \neq 0,\right. \\
\left.E_{\beta}^{\prime}(\vec{u})\left(u_{1}^{ \pm}, 0\right)=0, \quad E_{\beta}^{\prime}(\vec{u})\left(0, u_{2}\right)=0\right\} .
\end{gathered}
$$

Let $\vec{u}=\left(u_{1}, u_{2}\right)$ be any a nontrivial solution of (1.2) with $u_{1}$ sign-changing. Without loss of generality we assume $u_{2}^{+} \neq 0$. Then by a similar argument as Lemma 3.4 , there exists $t_{1}^{ \pm}, t_{2}^{+} \in(0,1]$ such that

$$
\left(\sqrt{t_{1}^{+}} u_{1}^{+}-\sqrt{t_{1}^{-}} u_{1}^{-}, \sqrt{t_{2}^{+}} u_{2}^{+}\right) \in \mathcal{N}_{2,1, \beta},
$$

and so

$$
\begin{aligned}
E_{\beta}\left(u_{2,1}, u_{2,2}\right) & =c_{\beta, \delta_{2}}^{2,1} \leq E\left(\sqrt{t_{1}^{+}} u_{1}^{+}-\sqrt{t_{1}^{-}} u_{1}^{-}, \sqrt{t_{2}^{+}} u_{2}^{+}\right) \\
& \leq \frac{1}{4}\left(\left\|u_{1}\right\|_{\lambda_{1}}^{2}+\left\|u_{2}^{+}\right\|_{\lambda_{2}}^{2}\right) \leq E_{\beta}(\vec{u})
\end{aligned}
$$

Hence $\left(u_{2,1}, u_{2,2}\right)$ has the least energy among all nontrivial solutions whose first component changes sign. This completes the proof.

\section{Asymptotic behaviors and phase separation}

In this section, we study the limit behavior of solutions obtained above as $\beta \rightarrow$ $-\infty$. Fix any $k_{1}, k_{2} \in \mathbb{N}$ such that $k_{1} \geq 2$ and $k_{2} \geq 1$. Then by the arguments in above sections we know that, for any $\beta<0$, there exists $\delta_{\beta} \in\left(0,2^{-1 / 4}\right)$ and 
$\vec{u}_{\beta}=\left(u_{\beta, 1}, u_{\beta, 2}\right) \in H$ such that $\vec{u}_{\beta}$ is a nontrivial solution (either sign-changing or semi-nodal) of (1.2) with

$$
E_{\beta}\left(\vec{u}_{\beta}\right)=c_{\beta, \delta_{\beta}}^{k_{1}, k_{2}} \leq c^{k_{1}, k_{2}}<+\infty .
$$

Here $c^{k_{1}, k_{2}}$ is seen in Lemmas 2.9 and 4.6. Recall that

$$
E_{\beta}\left(\vec{u}_{\beta}\right)=\frac{1}{4}\left(\left\|u_{\beta, 1}\right\|_{\lambda_{1}}^{2}+\left\|u_{\beta, 2}\right\|_{\lambda_{2}}^{2}\right)
$$

we see that $\vec{u}_{\beta}$ are uniformly bounded in $H$. On the other hand, by Kato's inequality (see [15]) we have

$$
\Delta\left|u_{\beta, i}\right| \geq \Delta u_{\beta, i} \cdot \frac{u_{\beta, i}}{\left|u_{\beta, i}\right|} \quad \text { in }\left(H_{0}^{1}(\Omega)\right)^{\prime} .
$$

Recall that $\beta<0$, then it is easy to check that

$$
-\Delta\left|u_{\beta, i}\right|+\lambda_{i}\left|u_{\beta, i}\right| \leq \mu_{i}\left|u_{\beta, i}\right|^{3}, \quad\left|u_{\beta, i}\right| \in H_{0}^{1}(\Omega) .
$$

Hence by standard Moser iteration, we see that $u_{\beta, i}$ are uniformly bounded in $L^{\infty}(\Omega)$ for any $\beta<0$ and $i=1,2$. Moreover, by elliptic regularity theory it holds that $u_{\beta, i} \in C(\bar{\Omega}) \cap C^{2}(\Omega)$. The main result of this section is following.

Theorem 5.1. There exists a vector Lipschitz function $\vec{u}_{\infty}=\left(u_{\infty, 1}, u_{\infty, 2}\right) \in \widetilde{H}$ such that, up to a subsequence,

(1) $u_{\beta, i} \rightarrow u_{\infty, i}$ in $H_{0}^{1}(\Omega) \cap C^{0, \alpha}(\bar{\Omega})$ for every $0<\alpha<1$ as $\beta \rightarrow-\infty$;

(2) $-\Delta u_{\infty, i}+\lambda_{i} u_{\infty, i}=\mu_{i} u_{\infty, i}^{3}$ in the open set $\left\{u_{\infty, i} \neq 0\right\}$;

(3) $u_{\infty, 1} \cdot u_{\infty, 2} \equiv 0$ and $|\beta| \int_{\Omega} u_{\beta, 1}^{2} u_{\beta, 2}^{2} d x \rightarrow 0$ as $\beta \rightarrow-\infty$;

(4) if $k_{1}, k_{2} \geq 2$, then $u_{\infty, i}$ changes sign for $i=1,2$. Moreover, if $k_{1}=k_{2}=2$, then $\left\{u_{\infty, i} \neq 0\right\}$ has exactly two connected components, and $u_{\infty, i}$ is a least energy sign-changing solution of

$$
-\Delta u+\lambda_{i} u=\mu_{i} u^{3}, \quad u \in H_{0}^{1}\left(\left\{u_{\infty, i} \neq 0\right\}\right)
$$

for $i=1,2$;

(5) if $k_{1} \geq 2$ and $k_{2}=1$, then $u_{\infty, 1}$ changes sign, $\left\{u_{\infty, 1} \neq 0\right\}$ has at most $k_{1}$ connected components and $u_{\infty, 2}$ is positive in $\left\{u_{\infty, 2} \neq 0\right\}$. Moreover, if $\left(k_{1}, k_{2}\right)=(2,1)$, then $\left\{u_{\infty, 1} \neq 0\right\}$ has exactly two connected components, $u_{\infty, 1}$ is a least energy sign-changing solution of (5.1) for $i=1,\left\{u_{\infty, 2} \neq 0\right\}$ is connected, and $u_{\infty, 2}$ is a least energy solution of (5.1) for $i=2$.

Proof. Since $u_{\beta, i}$ are uniformly bounded in $L^{\infty}(\Omega)$ for any $\beta<0$ and $i=1,2$, then (1) - (3) follows from [26, Theorems 1.1 and 1.2]. Remark that, although in [26] the results are stated for nonnegative solutions, they also hold for solutions with no sign-restrictions; all arguments there can be adapted with little extra effort 
to this more general case, working with the positive and negative parts of a solution. This fact was pointed out in the proof of [29, Theorem 4.3].

It remains to prove (4) $-(5)$. First we consider the case $k_{1}, k_{2} \geq 2$. Since $u_{\beta, i} \in C(\bar{\Omega}) \cap C^{2}(\Omega)$ and $u_{\beta, i}$ changes sign, so there exists $x_{\beta, i}^{ \pm} \in \Omega$ such that

$$
u_{\beta, i}\left(x_{\beta, i}^{+}\right)=\max _{x \in \Omega} u_{\beta, i}(x)>0 \quad \text { and } \quad u_{\beta, i}\left(x_{\beta, i}^{-}\right)=\min _{x \in \Omega} u_{\beta, i}(x)<0, \quad i=1,2 .
$$

Then $\Delta u_{\beta, i}\left(x_{\beta, i}^{+}\right) \leq 0$. Since $\vec{u}_{\beta}$ satisfies (1.2) and $\beta<0$, so $\lambda_{i} u_{\beta, i}\left(x_{\beta, i}^{+}\right) \leq$ $\mu_{i} u_{\beta, i}^{3}\left(x_{\beta, i}^{+}\right)$, which implies

$$
u_{\beta, i}\left(x_{\beta, i}^{+}\right)=\max _{x \in \Omega} u_{\beta, i}(x) \geq \sqrt{\lambda_{i} / \mu_{i}}, \quad \forall \beta<0 .
$$

Similarly,

$$
u_{\beta, i}\left(x_{\beta, i}^{-}\right)=\min _{x \in \Omega} u_{\beta, i}(x) \leq-\sqrt{\lambda_{i} / \mu_{i}}, \quad \forall \beta<0 .
$$

Combining these with (1), we see that $u_{\infty, i}$ changes sign, and so $\left\{u_{\infty, i} \neq 0\right\}$ has at least two connected components.

Now we let $\left(k_{1}, k_{2}\right)=(2,2)$. Assume by contradiction that $\left\{u_{\infty, 1} \neq 0\right\}$ has at least three connected components $\Omega_{1}, \Omega_{2}$ and $\Omega_{3}$. Without loss of generality, we assume that $u_{\infty, 1}>0$ on $\Omega_{1} \cup \Omega_{2}$. As in the proof of Lemma 3.4, we define $u_{1}^{+}:=$ $\chi \Omega_{\Omega_{1}} u_{\infty, 1}, u_{1}^{-}:=\chi \chi_{\Omega_{2}} u_{\infty, 1}$ and $u_{3}:=\chi_{\Omega_{3}} u_{\infty, 1}$, then $\left\|u_{\infty, 1}\right\|_{\lambda_{1}}^{2}>\left\|u_{1}^{+}\right\|_{\lambda_{1}}^{2}+\left\|u_{1}^{-}\right\|_{\lambda_{1}}^{2}$. By Theorem 5.1-(2) and (3.3) it is easy to see that $\left(u_{1}^{+}-u_{1}^{-}, u_{\infty, 2}\right) \in \mathcal{N}_{\beta}$ for all $\beta<0$, so

$$
\begin{aligned}
\frac{1}{4}\left(\left\|u_{\infty, 1}\right\|_{\lambda_{1}}^{2}+\left\|u_{\infty, 2}\right\|_{\lambda_{2}}^{2}\right) & =\lim _{\beta \rightarrow-\infty} \frac{1}{4}\left(\left\|u_{\beta, 1}\right\|_{\lambda_{1}}^{2}+\left\|u_{\beta, 2}\right\|_{\lambda_{2}}^{2}\right) \\
& =\lim _{\beta \rightarrow-\infty} E_{\beta}\left(u_{\beta, 1}, u_{\beta, 2}\right)=\lim _{\beta \rightarrow-\infty} c_{\beta, \delta_{\beta}}^{2,2} \\
& \leq \lim _{\beta \rightarrow-\infty} E_{\beta}\left(u_{1}^{+}-u_{1}^{-}, u_{\infty, 2}\right) \\
& =\frac{1}{4}\left(\left\|u_{1}^{+}\right\|_{\lambda_{1}}^{2}+\left\|u_{1}^{-}\right\|_{\lambda_{1}}^{2}+\left\|u_{\infty, 2}\right\|_{\lambda_{2}}^{2}\right)
\end{aligned}
$$

a contradiction. Hence $\left\{u_{\infty, i} \neq 0\right\}$ has exactly two connected components. If $u_{i} \in H_{0}^{1}\left(\left\{u_{\infty, i} \neq 0\right\}\right)$ is any sign-changing solutions of $(5.1)$, then $\left(u_{1}, u_{\infty, 2}\right)$, $\left(u_{\infty, 1}, u_{2}\right) \in \mathcal{N}_{\beta}$ for all $\beta<0$, so similarly as (5.2) we see that $\left\|u_{\infty, i}\right\|_{\lambda_{i}}^{2} \leq\left\|u_{i}\right\|_{\lambda_{i}}^{2}$, that is, $u_{\infty, i}$ has the least energy among all sign-changing solutions of (5.1). Hence $u_{\infty, i}$ is a least energy sign-changing solution of (5.1), and (4) holds.

Now we consider $k_{1} \geq 2$ and $k_{2}=1$. Then $u_{\infty, 1}$ changes sign as above. Since $u_{\beta, 2}$ is positive, so $u_{\infty, 2}>0$ in $\left\{u_{\infty, 2} \neq 0\right\}$. Define

$$
\begin{aligned}
\mathcal{N}_{k_{1}, 1, \beta}:= & \left\{\vec{u}=\left(u_{1}, u_{2}\right) \in H: u_{1} \text { changes sign and has at least } k_{1}\right. \text { nodal } \\
& \text { domains } \Omega_{k}\left(1 \leq k \leq k_{1}\right), u_{2} \neq 0, u_{2} \geq 0, \\
& \left.E_{\beta}^{\prime}(\vec{u})\left(u_{1} \chi_{\Omega_{k}}, 0\right)=0, \forall 1 \leq k \leq k_{1}, E_{\beta}^{\prime}(\vec{u})\left(0, u_{2}\right)=0\right\} .
\end{aligned}
$$


Then the same arguments as (4.14)-(4.16) yield that

$$
c_{\beta, \delta_{\beta}}^{k_{1}, 1} \leq \inf _{\vec{u} \in \mathcal{N}_{k_{1}, 1, \beta}} E_{\beta}(\vec{u})
$$

If $\left\{u_{\infty, 1} \neq 0\right\}$ has at least $k_{1}+1$ connected components $\Omega_{k}\left(1 \leq k \leq k_{1}+1\right)$, then

$$
\left(u_{\infty, 1} \chi_{\cup_{k=1}^{k_{1}} \Omega_{k}}, u_{\infty, 2}\right) \in \mathcal{N}_{k_{1}, 1, \beta} \quad \forall \beta<0 .
$$

Similarly as (5.2) we get a contradiction. Hence, $\left\{u_{\infty, 1} \neq 0\right\}$ has at most $k_{1}$ connected components.

If $\left(k_{1}, k_{2}\right)=(2,1)$, then $\left\{u_{\infty, 1} \neq 0\right\}$ has exactly two connected components. If $\left\{u_{\infty, 2} \neq 0\right\}$ has at least two connected components $\Omega_{1}$ and $\Omega_{2}$, then $\left(u_{\infty, 1}, u_{\infty, 2} \chi_{\Omega_{1}}\right) \in \mathcal{N}_{2,1, \beta}$ for all $\beta<0$, and similarly as (5.2) we get a contradiction. Hence $\left\{u_{\infty, 2} \neq 0\right\}$ is connected. Finally, similarly as above, we can prove that $u_{\infty, 1}$ is a least energy sign-changing solution of (5.1) for $i=1$, and $u_{\infty, 2}$ is a least energy solution of (5.1) for $i=2$. This completes the proof.

\section{The entire space case}

In this final section, we extend some results above to the case where $\Omega=\mathbb{R}^{N}$. That is, we consider the following elliptic system in the entire space

$$
\begin{cases}-\Delta u_{1}+\lambda_{1} u_{1}=\mu_{1} u_{1}^{3}+\beta u_{1} u_{2}^{2}, & x \in \mathbb{R}^{N}, \\ -\Delta u_{2}+\lambda_{2} u_{2}=\mu_{2} u_{2}^{3}+\beta u_{1}^{2} u_{2}, & x \in \mathbb{R}^{N}, \\ u_{1}(x), u_{2}(x) \rightarrow 0 \text { as }|x| \rightarrow+\infty . & \end{cases}
$$

By giving some modifications to arguments in Sections 2-4 and introducing some different ideas and techniques, we can prove the following results.

Theorem 6.1. Let $N=2,3, \lambda_{1}, \lambda_{2}, \mu_{1}, \mu_{2}>0$ and $\beta<0$. Then (6.1) has infinitely many radially symmetric sign-changing solutions, including a special $\left(u_{1}, u_{2}\right)$ such that both $u_{1}$ and $u_{2}$ have exactly two nodal domains and $\left(u_{1}, u_{2}\right)$ has the least energy among all radially symmetric sign-changing solutions.

Theorem 6.2. Let assumptions in Theorem 6.1 hold. Then (6.1) has infinitely many radially symmetric semi-nodal solutions $\left\{\left(u_{n, 1}, u_{n, 2}\right)\right\}_{n \geq 2}$ such that

(1) $u_{n, 1}$ changes sign and $u_{n, 2}$ is positive;

(2) $u_{n, 1}$ has at most $n$ nodal domains. In particular, $u_{2,1}$ has exactly two nodal domains, and $\left(u_{2,1}, u_{2,2}\right)$ has the least energy among all nontrivial radially symmetric solutions whose first component changes sign. 
Remark 6.3. Let assumptions in Theorem 6.1 hold. Lin and Wei [16] proved that (6.1) has no least energy solutions. Later, Sirakov [27] proved that (6.1) has a radially symmetric positive solution which has the least energy among all nontrivial radially symmetric solutions. Combining these with the introduction in Section 1, our results here are completely new.

Define $H_{r}:=H_{r}^{1}\left(\mathbb{R}^{N}\right) \times H_{r}^{1}\left(\mathbb{R}^{N}\right)$ as a subspace of $H:=H^{1}\left(\mathbb{R}^{N}\right) \times H^{1}\left(\mathbb{R}^{N}\right)$ with norm $\left\|\left(u_{1}, u_{2}\right)\right\|_{H}^{2}=\left\|u_{1}\right\|_{\lambda_{1}}^{2}+\left\|u_{2}\right\|_{\lambda_{2}}^{2}$, where

$$
\begin{aligned}
& H_{r}^{1}\left(\mathbb{R}^{N}\right):=\left\{u \in H^{1}\left(\mathbb{R}^{N}\right): u \text { is radially symmetric }\right\}, \\
& \|u\|_{\lambda_{i}}^{2}:=\int_{\mathbb{R}^{N}}\left(|\nabla u|^{2}+\lambda_{i} u^{2}\right) d x .
\end{aligned}
$$

Since the embedding $H_{r}^{1}\left(\mathbb{R}^{N}\right) \hookrightarrow L^{4}\left(\mathbb{R}^{N}\right)$ is compact, by replacing $H_{0}^{1}(\Omega), H$ with $H_{r}^{1}\left(\mathbb{R}^{N}\right), H_{r}$ respectively in all definitions appeared in Sections 2-4 and using the same notations, it is easy to see that all arguments (with trivial modifications) in Sections 2-4 hold for system (6.1) except those in Step 2 of proving Theorems 1.3 and 1.7. Hence we only need to reprove Step 2 in the proofs of Theorems 1.3 and 1.7. The following ideas and arguments are quite different from those in Step 2 of proving Theorems 1.3 and 1.7, and also can be used in the bounded domain case.

Proof of Theorem 6.1. Assume by contradiction that there exists $n_{0} \in \mathbb{N}$ such that (6.1) has only $n_{0}$ radially symmetric sign-changing solutions. Fix any $k_{2} \geq 2$, we define

$$
l:=\max \left\{c^{k_{1}, k_{2}}: 2 \leq k_{1} \leq n_{0}+2\right\}+1 \text {. }
$$

For any $k_{1} \in\left[2, n_{0}+2\right]$ and $0<\delta<2^{-1 / 4}$, similarly as (2.22)-(2.23) we define

$$
c_{\beta, l, \delta}^{k_{1}, k_{2}}:=\inf _{A \in \Gamma_{\beta, l}^{\left(k_{1}, k_{2}\right)}} \sup _{\vec{u} \in A \backslash \mathcal{P}_{\delta}} J_{\beta}(\vec{u}),
$$

where

$$
\Gamma_{\beta, l}^{\left(k_{1}, k_{2}\right)}:=\left\{A \in \Gamma^{\left(k_{1}, k_{2}\right)}: \sup _{A} J_{\beta}<l\right\} .
$$

Lemma 2.9 yields that $\Gamma_{\beta, l}^{\left(k_{1}, k_{2}\right)} \neq \emptyset, c_{\beta, l, \delta}^{k_{1}, k_{2}}$ is well defined and $c_{\beta, l, \delta}^{k_{1}, k_{2}} \leq c^{k_{1}, k_{2}}$ for each $k_{1} \in\left[2, n_{0}+2\right]$. Noting that $\Gamma_{\beta, l}^{\left(k_{1}+1, k_{2}\right)} \subset \Gamma_{\beta, l}^{\left(k_{1}, k_{2}\right)}$, we have

$$
c_{\beta, l, \delta}^{2, k_{2}} \leq c_{\beta, l, \delta}^{3, k_{2}} \leq \cdots \leq c_{\beta, l, \delta}^{n_{0}+1, k_{2}} \leq c_{\beta, l, \delta}^{n_{0}+2, k_{2}}
$$

By repeating the arguments in Section 2 we can prove that there exists $\delta_{l} \in\left(0,2^{-1 / 4}\right)$ such that, for any $\delta \in\left(0, \delta_{l}\right)$,

$$
\eta(t, \vec{u}) \in \mathcal{P}_{\delta} \quad \text { whenever } u \in \mathcal{M}_{\beta} \cap \mathcal{P}_{\delta}, J_{\beta}(u) \leq l \text { and } t>0,
$$


and so $c_{\beta, l, \delta}^{k_{1}, k_{2}}$ is a radially symmetric sign-changing critical value of $E_{\beta}$ for each $k_{1} \in\left[2, n_{0}+2\right]$ (that is, $E_{\beta}$ has a radially symmetric sign-changing critical point $\vec{u}$ with $\left.E_{\beta}(\vec{u})=c_{\beta, l, \delta}^{k_{1}, k_{2}}\right)$. Fix any a $\delta \in\left(0, \delta_{l}\right)$. By (6.4) and our assumption that (6.1) has only $n_{0}$ radially symmetric sign-changing solutions, there exists some $2 \leq N_{1} \leq n_{0}+1$ such that

$$
c_{\beta, l, \delta}^{N_{1}, k_{2}}=c_{\beta, l, \delta}^{N_{1}+1, k_{2}}=: \bar{c} .
$$

Define

$$
\mathcal{K}:=\left\{\vec{u} \in \mathcal{M}: \vec{u} \text { is sign-changing, } \quad J_{\beta}(\vec{u})=\bar{c}, \quad V(\vec{u})=0\right\} .
$$

Then $\mathcal{K}$ is finite. By (2.16) one has that $\sigma_{i}(\vec{u}) \in \mathcal{K}$ if $\vec{u} \in \mathcal{K}$, that is, $\mathcal{K} \subset \mathcal{F}$. Hence there exist $k \leq n_{0}$ and $\left\{\vec{u}_{m}: 1 \leq m \leq k\right\} \subset \mathcal{K}$ such that

$$
\mathcal{K}=\left\{\vec{u}_{m}, \sigma_{1}\left(\vec{u}_{m}\right), \sigma_{2}\left(\vec{u}_{m}\right),-\vec{u}_{m}: 1 \leq m \leq k\right\}
$$

Then there exist open neighborhoods $O_{\vec{u}_{m}}$ of $\vec{u}_{m}$ in $H_{r}$, such that any two of $\overline{O_{\vec{u}_{m_{1}}}}, \sigma_{1}\left(\overline{O_{\vec{u}_{m_{2}}}}\right), \sigma_{2}\left(\overline{O_{\vec{u}_{m_{3}}}}\right)$ and $-\overline{O_{\vec{u}_{m_{4}}}}$, where $1 \leq m_{1}, m_{2}, m_{3}, m_{4} \leq k$, are disjointed and

$$
\mathcal{K} \subset O:=\bigcup_{m=1}^{k} O_{\vec{u}_{m}} \cup \sigma_{1}\left(O_{\vec{u}_{m}}\right) \cup \sigma_{2}\left(O_{\vec{u}_{m}}\right) \cup-O_{\vec{u}_{m}}
$$

Define a continuous map $\tilde{f}: \bar{O} \rightarrow \mathbb{R} \backslash\{0\}$ by

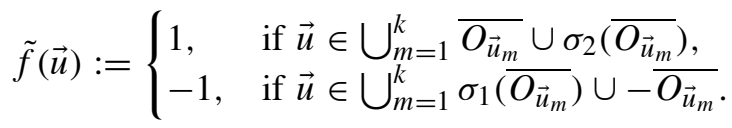

Then $\tilde{f}\left(\sigma_{1}(\vec{u})\right)=-\tilde{f}(\vec{u})$ and $\tilde{f}\left(\sigma_{2}(\vec{u})\right)=\tilde{f}(\vec{u})$. By Tietze's extension theorem, there exists $f \in C\left(H_{r}, \mathbb{R}\right)$ such that $\left.f\right|_{O} \equiv \tilde{f}$. Define

$$
F(\vec{u}):=\frac{f(\vec{u})+f\left(\sigma_{2}(\vec{u})\right)-f\left(\sigma_{1}(\vec{u})\right)-f(-\vec{u})}{4},
$$

then $\left.F\right|_{O} \equiv \tilde{f}, F\left(\sigma_{1}(\vec{u})\right)=-F(\vec{u})$ and $F\left(\sigma_{2}(\vec{u})\right)=F(\vec{u})$. Define

$$
\mathcal{K}_{\tau}:=\left\{\vec{u} \in \mathcal{M}: \inf _{\vec{v} \in \mathcal{K}}\|\vec{u}-\vec{v}\|_{H}<\tau\right\} .
$$

Then we can take small $\tau>0$ such that $\mathcal{K}_{2 \tau} \subset O$. Recalling $V(\vec{u})=0$ in $\mathcal{K}$ and $\mathcal{K}$ finite, there exists $\widetilde{C}>0$ such that

$$
\|V(\vec{u})\|_{H} \leq \widetilde{C}, \quad \forall \vec{u} \in \overline{\mathcal{K}_{2 \tau}} .
$$


For any $\vec{u} \in \mathcal{K}_{2 \tau}$, we have $F(\vec{u})=\tilde{f}(\vec{u}) \neq 0$. That is $F\left(\mathcal{K}_{2 \tau}\right) \subset \mathbb{R} \backslash\{0\}$. By (6.7) and Lemma 2.11 there exists small $\varepsilon \in(0,1)$ such that

$$
\|V(\vec{u})\|_{H}^{2} \geq \varepsilon, \quad \forall u \in \mathcal{M}_{\beta} \backslash\left(\mathcal{K}_{\tau} \cup \mathcal{P}_{\delta}\right) \text { satisfying }\left|J_{\beta}(\vec{u})-\bar{c}\right| \leq 2 \varepsilon .
$$

Recall $C_{0}$ in (2.17), we let

$$
\alpha:=\frac{1}{2} \min \left\{1, \frac{\tau C_{0}}{2 \widetilde{C}}\right\} .
$$

By (6.2)-(6.3) and (6.6) we take $A \in \Gamma_{\beta, l}^{\left(N_{1}+1, k_{2}\right)}$ such that

$$
\sup _{A \backslash \mathcal{P}_{\delta}} J_{\beta}<c_{\beta, l, \delta}^{N_{1}+1, k_{2}}+\alpha \varepsilon=\bar{c}+\alpha \varepsilon
$$

Let $B:=A \backslash \mathcal{K}_{2 \tau}$, then it is easy to check that $B \subset \mathcal{F}$. We claim that $\vec{\gamma}(B) \geq$ $\left(N_{1}, k_{2}\right)$. If not, there exists $\tilde{g} \in F_{\left(N_{1}, k_{2}\right)}(B)$ such that $\tilde{g}(\vec{u}) \neq 0$ for any $\vec{u} \in \vec{B}$. By Tietze's extension theorem, there exists $\bar{g}=\left(\bar{g}_{1}, \bar{g}_{2}\right) \in C\left(H_{r}, \mathbb{R}^{N_{1}-1} \times \mathbb{R}^{k_{2}-1}\right)$ such that $\left.\bar{g}\right|_{B} \equiv \tilde{g}$. Define $g=\left(g_{1}, g_{2}\right) \in C\left(H_{r}, \mathbb{R}^{N_{1}-1} \times \mathbb{R}^{k_{2}-1}\right)$ by

$$
\begin{aligned}
& g_{1}(\vec{u}):=\frac{\bar{g}_{1}(\vec{u})+\bar{g}_{1}\left(\sigma_{2}(\vec{u})\right)-\bar{g}_{1}\left(\sigma_{1}(\vec{u})\right)-\bar{g}_{1}(-\vec{u})}{4}, \\
& g_{2}(\vec{u}):=\frac{\bar{g}_{2}(\vec{u})+\bar{g}_{2}\left(\sigma_{1}(\vec{u})\right)-\bar{g}_{2}\left(\sigma_{2}(\vec{u})\right)-\bar{g}_{2}(-\vec{u})}{4},
\end{aligned}
$$

then $\left.g\right|_{B} \equiv \tilde{g}, g_{i}\left(\sigma_{i}(\vec{u})\right)=-g_{i}(\vec{u})$ and $g_{i}\left(\sigma_{j}(\vec{u})\right)=g_{i}(\vec{u})$ for $j \neq i$. Finally we define $G=\left(G_{1}, G_{2}\right) \in C\left(A, \mathbb{R}^{N_{1}+1-1} \times \mathbb{R}^{k_{2}-1}\right)$ by

$$
G_{1}(\vec{u}):=\left(F(\vec{u}), g_{1}(\vec{u})\right) \in \mathbb{R}^{N_{1}+1-1}, \quad G_{2}(\vec{u}):=g_{2}(\vec{u}) \in \mathbb{R}^{k_{2}-1} .
$$

By our constructions of $F$ and $g$, we have $G \in F_{\left(N_{1}+1, k_{2}\right)}(A)$. Since $\vec{\gamma}(A) \geq$ $\left(N_{1}+1, k_{2}\right)$, so $G(\vec{u})=0$ for some $\vec{u} \in A$. If $\vec{u} \in \mathcal{K}_{2 \tau}$, then $F(\vec{u}) \neq 0$, a contradiction. So $\vec{u} \in A \backslash \mathcal{K}_{2 \tau}=B$, and then $g(\vec{u})=\tilde{g}(\vec{u}) \neq 0$, also a contradiction. Hence $\vec{\gamma}(B) \geq\left(N_{1}, k_{2}\right)$. Note that $\sup _{B} J_{\beta} \leq \sup _{A} J_{\beta}<l$, we see that $B \subset \mathcal{M}_{\beta}$ and $B \in \Gamma_{\beta, l}^{\left(N_{1}, k_{2}\right)}$. Then we can consider $D:=\eta(\tau /(2 \widetilde{C}), B)$, where $\eta$ is in Lemma 2.13 and $\widetilde{C}$ is in (6.8). By Lemma 2.7-(ii) and Lemma 2.13 we have $D \in \Gamma^{\left(N_{1}, k_{2}\right)}$ and $\sup _{D} J_{\beta} \leq \sup _{B} J_{\beta}<l$, that is $D \in \Gamma_{\beta, l}^{\left(N_{1}, k_{2}\right)}$. Then we see from (6.2)-(6.3) and (6.6) that

$$
\sup _{D \backslash \mathcal{P}_{\delta}} J_{\beta} \geq c_{\beta, l, \delta}^{N_{1}, k_{2}}=\bar{c}
$$

By Lemma 2.8 we can take $\vec{u} \in B$ such that $\eta(\tau /(2 \widetilde{C}), \vec{u}) \in D \backslash \mathcal{P}_{\delta}$ and

$$
\bar{c}-\alpha \varepsilon \leq \sup _{D \backslash \mathcal{P}_{\delta}} J_{\beta}-\alpha \varepsilon<J_{\beta}(\eta(\tau /(2 \widetilde{C}), \vec{u})) .
$$


Since $J_{\beta}(\eta(t, \vec{u})) \leq J_{\beta}(\vec{u})<l$ for any $t \geq 0$, (6.5) yields $\eta(t, \vec{u}) \notin \mathcal{P}_{\delta}$ for any $t \in[0, \tau /(2 \widetilde{C})]$. In particular, $\vec{u} \notin \mathcal{P}_{\delta}$ and so (6.11) yields $J_{\beta}(\vec{u})<\bar{c}+\alpha \varepsilon$. Then for any $t \in[0, \tau /(2 \widetilde{C})]$, we have

$$
\bar{c}-\alpha \varepsilon<J_{\beta}(\eta(\tau /(2 \widetilde{C}), \vec{u})) \leq J_{\beta}(\eta(t, \vec{u})) \leq J_{\beta}(\vec{u})<\bar{c}+\alpha \varepsilon .
$$

Recall that $\vec{u} \in B=A \backslash \mathcal{K}_{2 \tau}$. If there exists $T \in(0, \tau /(2 \widetilde{C}))$ such that $\eta(T, \vec{u}) \in$ $\mathcal{K}_{\tau}$, then there exist $0 \leq t_{1}<t_{2} \leq T$ such that $\eta\left(t_{1}, \vec{u}\right) \in \partial \mathcal{K}_{2 \tau}, \eta\left(t_{2}, \vec{u}\right) \in \partial \mathcal{K}_{\tau}$ and $\eta(t, \vec{u}) \in \mathcal{K}_{2 \tau} \backslash \mathcal{K}_{\tau}$ for any $t \in\left(t_{1}, t_{2}\right)$. So we see from (6.8) that

$$
\tau \leq\left\|\eta\left(t_{1}, \vec{u}\right)-\eta\left(t_{2}, \vec{u}\right)\right\|_{H}=\left\|\int_{t_{1}}^{t_{2}} V(\eta(t, \vec{u})) d t\right\|_{H} \leq 2 \widetilde{C}\left(t_{2}-t_{1}\right),
$$

that is, $\tau /(2 \widetilde{C}) \leq t_{2}-t_{1} \leq T$, a contradiction. Hence $\eta(t, \vec{u}) \notin \mathcal{K}_{\tau}$ for any $t \in(0, \tau /(2 \widetilde{C}))$. Then as Step 1 in the proof of Theorem 1.3, we deduce from (6.9) and (6.10) that

$$
\bar{c}-\alpha \varepsilon<J_{\beta}(\eta(\tau /(2 \widetilde{C}), \vec{u})) \leq J_{\beta}(\vec{u})-\int_{0}^{\tau /(2 \widetilde{C})} C_{0} \varepsilon d t<\bar{c}+\alpha \varepsilon-2 \alpha \varepsilon=\bar{c}-\alpha \varepsilon,
$$

a contradiction. Hence (6.1) has infinite many radially symmetric sign-changing solutions. This completes the proof.

Proof of Theorem 6.2. It suffices to prove that (6.1) has infinitely many semi-nodal solutions. This argument is similar as above, we omit the details.

\section{References}

[1] N. AKHMEDIEV and A. ANKIEWICZ, Partially coherent solitons on a finite background, Phys. Rev. Lett. 82 (1999), 2661-2664.

[2] A. Ambrosetti and E. Colorado, Standing waves of some coupled nonlinear Schrödinger equations, J. Lond. Math. Soc. 75 (2007), 67-82.

[3] T. BARTSCH, N. DANCER and Z.-Q. WANG, A Liouville theorem, a priori bounds, and bifurcating branches of positive solutions for a nonlinear elliptic system, Calc. Var. Partial Differential Equations 37 (2010), 345-361.

[4] T. BARTSCH, Z. LIU and T. WETH, Sign changing solutions of superlinear Schrödinger equations, Comm. Partial Differential Equations 29 (2004), 25-42.

[5] T. BARTSCH and Z.-Q. WANG, Note on ground states of nonlinear Schrödinger systems, J. Partial Differential Equations 19 (2006), 200-207.

[6] T. BARTSCH, Z.-Q. WANG and J. WEI, Bound states for a coupled Schrödinger system, J. Fixed Point Theory Appl. 2 (2007), 353-367.

[7] L. A. CAFFARELLI and F.-H. LIN, Singularly perturbed elliptic systems and multi-valued harmonic functions with free boundaries, J. Amer. Math. Soc. 21 (2008), 847-862.

[8] S. Chang, C. Lin, T. Lin and W. Lin, Segregated nodal domains of two-dimensional multispecies Bose-Einstein condensates, Phys. D. 196 (2004), 341-361. 
[9] Z. CHEN and W. Zou, Positive least energy solutions and phase separation for coupled Schrödinger equations with critical exponent, Arch. Ration. Mech. Anal. 205 (2012), 515551.

[10] Z. CHEN and W. Zou, An optimal constant for the existence of least energy solutions of a coupled Schrödinger system, Calc. Var. Partial Differential Equations 48 (2013), 695-711.

[11] M. CONTI, L. MERIZZI and S. TERRACINI, Remarks on variational methods and lowerupper solutions, Nonlinear Differential Equations Appl. 6 (1999), 371-393.

[12] M. CONTI, S. TERRACINI and G. VERZINI, Asymptotic estimates for the spatial segregation of competitive systems, Adv. Math. 195 (2005), 524-560.

[13] N. DANCER, J. WEI and T. WETH, A priori bounds versus multiple existence of positive solutions for a nonlinear Schrödinger system, Ann. Inst. H. Poincaré, Anal. Non Linéaire 27 (2010), 953-969.

[14] B. Esry, C. Greene, J. Burke and J. Bohn, Hartree-Fock theory for double condensates, Phys. Rev. Lett. 78 (1997), 3594-3597.

[15] T. KATO, Schrödinger operators with singular potentials, Israel J. Math. 13 (1972), 135148.

[16] T. LIN and J. WEI, Ground state of $N$ coupled nonlinear Schrödinger equations in $\mathbb{R}^{n}$, $n \leq 3$, Comm. Math. Phys. 255 (2005), 629-653.

[17] T. LIN and J. WEI, Spikes in two coupled nonlinear Schrödinger equations, Ann. Inst. H. Poincaré, Anal. Non Linéaire 22 (2005), 403-439.

[18] J. LiU, X. LIU and Z.-Q. WANG, Multiple mixed states of nodal solutions for nonlinear Schrödinger systems, Calc. Var. Partial Differential Equations 52 (2015), 565-586.

[19] Z. LIU and Z.-Q. WANG, Multiple bound states of nonlinear Schrödinger systems, Comm. Math. Phys. 282 (2008), 721-731.

[20] Z. LIU and Z.-Q. WANG, Ground states and bound states of a nonlinear Schrödinger system, Adv. Nonlinear Stud. 10 (2010), 175-193.

[21] L. Maia, E. Montefusco and B. PellacCI, Positive solutions for a weakly coupled nonlinear Schrödinger system, J. Differential Equations 229 (2006), 743-767.

[22] L. Maia, E. Montefusco and B. PellacCI, Infinitely many nodal solutions for a weakly coupled nonlinear Schrödinger system, Commun. Contemp. Math. 10 (2008), 651669.

[23] C. Miranda, Un'osservazione su un teorema di Brouwer, Boll. Unione Mat. Ital. II Ser. 3 (1940), 5-7.

[24] B. NORIS and M. RAMOS, Existence and bounds of positive solutions for a nonlinear Schrödinger system, Proc. Amer. Math. Soc. 138 (2010), 1681-1692.

[25] B. Noris, H. TAVAres, S. Terracini and G. Verzini, Convergence of minimax and continuation of critical points for singularly perturbed systems, J. Eur. Math. Soc. (JEMS) 14 (2012), 1245-1273.

[26] B. Noris, H. Tavares, S. Terracini and G. Verzini, Uniform Hölder bounds for nonlinear Schrödinger systems with strong competition, Comm. Pure Appl. Math. 63 (2010), 267-302.

[27] B. SIRAKOV, Least energy solitary waves for a system of nonlinear Schrödinger equations in $\mathbb{R}^{n}$, Comm. Math. Phys. 271 (2007), 199-221.

[28] Y. S ATO and Z. WANG, On the multiple existence of semi-positive solutions for a nonlinear Schrödinger system, Ann. Inst. H. Poincaré, Anal. Non Linéaire 30 (2013), 1-22.

[29] H. TAVARES and S. TERRACINI, Sign-changing solutions of competition-diffusion elliptic systems and optimal partition problems, Ann. Inst. H. Poincaré, Anal. Non Linéaire 29 (2012), 279-300.

[30] H. TAVARES and S. TERRACINI, Regularity of the nodal set of segregated critical configurations under a weak reflection law, Calc. Var. Partial Differential Equations 45 (2012), 273-317.

[31] S. TERRACINI and G. Verzini, Multipulse phases in k-mixtures of Bose-Einstein condensates, Arch. Ration. Mech. Anal. 194 (2009), 717-741. 
[32] J. WEI and T. WETH, Radial solutions and phase separation in a system of two coupled Schrödinger equations, Arch. Ration. Mech. Anal. 190 (2008), 83-106.

[33] J. WEI and T. WETH, Asymptotic behaviour of solutions of planar elliptic systems with strong competition, Nonlinearity 21 (2008), 305-317.

[34] W. Zou, "Sign-changing Critical Points Theory", Springer-New York, 2008.

Department of Mathematical Sciences

Tsinghua University

Beijing 100084, China

chenzhijie1987@sina.com

wzou@math.tsinghua.edu.cn

Taida Institute for Mathematical Sciences

Center for Advanced Study in

Theoretical Science

National Taiwan University

Taipei 106, Taiwan

cslin@math.ntu.edu.tw 